\title{
Entrepreneurial skills and wage employment
}

\author{
Aleksander Kucel \\ Escola Superior de Ciències Socials i de l'Empresa TecnoCampus, \\ Universitat Pompeu Fabra \\ $\&$ \\ CREB, Universitat de Barcelona \\ Montserrat Vilalta-Bufi* \\ Departament de Teoria Economica and CREB, \\ Universitat de Barcelona
}

July 2015

Corresponding Author: Montserrat Vilalta-Bufí, email: montsevilalta@ub.edu, tel. +34934024572, Departament de Teoria Econòmica and CREB, Universitat de Barcelona, Avda. Diagonal 696, 08034 Barcelona (Spain).

\footnotetext{
* Montserrat Vilalta-Bufí gratefully acknowledges financial support from the Spanish Ministry of Science and Innovation through the grant ECO2012-34046. The authors also wish to thank the financial support of the Government of Catalonia through the Barcelona GSE Research Network and grant 2014SGR_493 and the REFLEX project for granting access to their data.
} 


\begin{abstract}
Promotion of entrepreneurial skills among the population is often considered as an adequate policy to enhance job creation and economic growth. However, neither the definition of entrepreneurial skills, nor the costs and benefits of such a policy are clear. Our aim is to check whether the benefits of entrepreneurial skills extent beyond self-employment. We use a sample of higher education graduates from Spain, from the year 2000 interviewed in 2005 (REFLEX survey). We denote entrepreneurial skills as those competencies that enhance the likelihood of self-employment. Then we analyze whether they are rewarded in wage employment. We find that alertness to new opportunities, ability to mobilize others and knowledge of other fields are the competencies that enhance self-employment in Spain. Yet, these skills are not rewarded in a salaried job. Therefore, benefits of policies fostering entrepreneurial skills do not extend to wage employment in Spain.
\end{abstract}

Keywords: entrepreneurial skills; wage returns; wage employment; self-employment; competencies

JEL Codes: J24, J31, J43 


\section{Introduction}

Entrepreneurship has been continuously attracting all the more attention from both political elites (European Commission, 2009, European Commission, 2013) as well as the scientific community (Kuratko, 2005, Praag and Versloot, 2007, van Der Sluis et al., 2008). It gained interest in the context of the current economic crisis as it is commonly considered a motor of innovation and economic growth (Carlsson et al., 2009, Grossmann, 2009, Iyigun and Owen, 1999). Consequently, there is general support for policies directed to enhance the entrepreneurial competencies of the population. In this respect, entrepreneurial competencies have been added to the curricula of several studies, from secondary education to university degrees (Martin et al., 2013, Bae et al., 2014). However, within the literature on entrepreneurship it is not clear what entrepreneurship education should embrace (Béchard and Grégoire, 2005, Fiet, 2001, Haase and Lautenschläger, 2011, O'Connor, 2013), neither whether it has a positive effect on entrepreneurial activity. While some evidence points towards an increase in entrepreneurial success due to entrepreneurship education (Martin et al., 2013), other studies find a null or negative relationship (von Graevenitz et al., 2010, Oosterbeek et al., 2010 Bae et al., 2014).

The objective of this paper is twofold. First, we want to identify which competencies are positively correlated with self-employment in Spain. Second, we study whether these competencies are rewarded in wage employment. Although a general definition of entrepreneurship includes other than business founders and self-employed individuals, the literature has mostly considered entrepreneurship within the context of self-employment. We contribute to this literature by extending the analysis to wage employment. ${ }^{1}$ If entrepreneurial competencies ${ }^{2}$ were rewarded in wage employment, the benefits of policies promoting them would go beyond their intended effects on selfemployment. In contrast, if we find a non-positive effect on employees' wage, their potential benefits might have been over-estimated given that self-employment represents only a small proportion of the population. Finally, we check whether entrepreneurial skills are rewarded in self-employment.

Most papers on entrepreneurship agree that the core of entrepreneurial skills is the ability to create or recognize opportunities. There are different theories trying to discern how this process works. The most commonly accepted version is based on Kirzner's theory of alertness to new opportunities (Kirzner, 1973). Some authors argue that differences in alertness to new opportunities are the result of having different schema and pattern recognition (Baron and Ensley, 2006, Baron, 2004, Valliere, 2013). Gaglio (2004), instead, emphasizes the role of mental simulation and counter-factual thinking to develop opportunity alertness. Alertness proposed initially by Kirzner has been extended to creativity and self-consciousness in other papers (Kirzner, 1999 p. 12, Tang et al., 2012). Another strand of the literature finds that a successful entrepreneur needs to have a balanced set of skills rather than being good at one particular skill (Bublitz and Noseleit, 2014, Hartog et al., 2010, Stuetzer et al., 2012, Stuetzer et al., 2013, Lazear, 2004). We consider both strands in our analysis.

We use the REFLEX survey data. It includes self-reported information on 19 competencies, which range from alertness to new opportunities and ability to use time efficiently through ability to negotiate effectively or to write reports, among others. Three of these competencies can be linked to the

\footnotetext{
${ }^{1}$ A growing body of literature on corporate entrepreneurship, entrepreneurial employees and intrapreneurship is studying those employees that are key players in the development of new business activities within their firm (Sharma and Chrisman, 1999, Pinchot, 1985, Martiarena, 2013). We differ from this literature in that we consider the role of entrepreneurial skills for the average worker of the organization.

${ }^{2}$ There are many definitions of entrepreneurship in the literature. We label the competencies that increase the probability of self-employment as 'entrepreneurial competencies'. They may encompass leadership skills, creativity or management skills, among other competencies, as long as they boost self-employment activity.
} 
entrepreneurial skills described in the literature. The level of competency on 'alertness to new opportunities' directly measures entrepreneurial skills as proposed by Kirzner (1973). The competency on 'ability to come up with new ideas and solutions' can be related to creativity (Kirzner, 1999). Finally, the competency 'willingness to question your own and others' ideas' might reflect the counterfactual thinking emphasized by Gaglio (2004). We also construct a variable measuring the variety of skills to account for the balanced set of skills theory.

We estimate a Heckman selection model with wages in a salaried job as the main dependent variable and working in wage employment versus self-employment as the selection equation. Results reveal which competencies increase the likelihood of self-employment and which competencies are rewarded in wage employment when controlling for selection into such jobs. We also examine whether the variety of skills matters for self-employment and wage employment. Finally, we test which competencies are rewarded in self-employment.

The paper is structured as follows. In the next section, we review the literature on entrepreneurial skills and returns to entrepreneurship. Then, we present the data and econometric methods employed in our analysis. In section 4, we present and discuss the results. In section 5, we conclude and derive policy implications.

\section{Literature review}

The literature on entrepreneurship education and skills has grown rapidly during the last two decades. However, there is still little agreement on what entrepreneurial skills are. The ability to create or recognize opportunities is in the core of entrepreneurial skills (Kirzner, 1973). There is less consensus to classify other competencies as entrepreneurial skills. Some authors include the ability to evaluate these opportunities (Tang et al., 2012, Ardichvili et al., 2003), others include social ability and networks (Aldrich and Kim, 2007), risk tolerance and other personality characteristics (Caliendo et al., 2014).

Another strand of literature emphasizes the need of a balanced set of skills to become an entrepreneur (Bublitz and Noseleit, 2014, Hartog et al., 2010, Stuetzer et al., 2012, Stuetzer et al., 2013). Following Lazear (2004), p. 208, this literature claims that "entrepreneurs must be jacks-ofall-trades to some extent (...) to make sure that the business does not fail". For instance, Oberschachtsiek (2012) finds that a combination of broad experience with competency in sales/business is important for self-employment duration. As the company grows in size, the necessity for specialization of labor increases, reducing the required set of skills of each worker.

Regarding the core competency, there are several theories trying to discern how the process of opportunity recognition works. Personality traits such as creativity and optimism, counter-factual thinking, prior knowledge, cognitive ability, social networks and passion are some of the factors that have been associated to entrepreneurial alertness (Gaglio, 2004, Granovetter, 1973, Arenius and Clercq, 2005, Cardon et al., 2013, Shane et al., 2003). Other authors talk about the importance of different mental schema and pattern recognition in explaining opportunity recognition (Baron and Ensley, 2006, Baron, 2004, Valliere, 2013). According to them, individuals analyze reality via schemas. The richness of these schemas, the way to associate them to events and the sensitivity to changes determine the likelihood to identify new opportunities.

Following this literature and in line with the human capital theory (Becker, 1993), we propose entrepreneurial skills to be considered part of the human capital stock. Therefore, we expect university graduates to receive a wage premium to entrepreneurial skills aside from their standard human capital components, such as education, or labor market experience (Hanushek and Zhang, $\underline{2009}$, Heckman et al., 2003, Mincer, 1974). 
To our best knowledge, there is no research on the returns to entrepreneurial skills in wage employment. Within the entrepreneurship literature, some papers study the decision to become an entrepreneur (Blanchflower and Oswald, 1998, Lazear, 2005, Poschke, 2013, Hartog et al., 2010, Arenius and Minniti, 2005, Doms et al., 2010). The external environment (resources and political and market forces) along with the individual characteristics (ability, risk-aversion, opportunity recognition, among others) often influence the decision to become self-employed (Shane et al., 2003, Estrin et al., 2013). Other papers study the earnings differentials between self-employment and wage employment (Hamilton, 2000, Hyytinen et al., 2013). Some of these studies compare wages of entrepreneurs to wages of employees in Europe and the US. They find that, controlling for human capital, entrepreneurs earn on average less than employees and their wage growth curves are flatter in Europe, whilst the converse was found true for the U.S. (van der Sluis and van Praag, 2004, van Der Sluis et al., 2008). Non-pecuniary rewards to self-employment are often necessary to explain the lower initial earnings and lower earnings growth in self-employment. Martínez et al. (2007) provide a descriptive analysis of higher education graduates' wages when in salaried employment, and compare them to wages of self-employed. In their study, entrepreneurs with university degree have higher incomes and more satisfaction from their jobs, even though they work longer hours than the employee graduates do. Finally, in a similar vein, Hamilton (2000) finds non-pecuniary returns to self-employment.

Aside from the entrepreneurship literature, our research is also close to the empirical labor economics of skills. Various papers from education economics and labor economics have demonstrated that skills

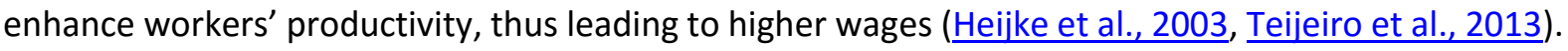
However, there is not yet a clear classification of skills as of now. As Teijeiro et al. (2013) note, the definition of skills used by each researcher depends mostly on data availability. Moreover, according to Biesma et al. (2007), as long as there is no common data collection framework, there will be no agreement on which skills are more important in the labor market. So far, most studies on returns to skills (either through wages, higher job satisfaction or higher on-the-job training possibilities) come mostly from small scale data projects (Allen and Velden, 2001, Allen and Van der Velden, 2009, GarciaAracil et al., 2004, Garcia-Aracil, 2009, Garcia-Aracil and Van der Velden, 2008, Mora et al., 2006). All this research clearly demonstrates that skills and, particularly what Heckman considers as "soft skills" (Heckman and Kautz, 2012), bring positive and significant returns in the labor market. Using CHEERS data (Career after Higher Education - A European Research Survey), Garcia-Aracil et al. (2004), for instance, show that what they call "participative" skills - which entail among others decision-making ability, assertiveness, and personal involvement - are the most rewarded in the European labor market. In another paper, also based on the CHEERS data, Garcia-Aracil and Van der Velden (2008) show that it is the jobs with higher "participative and methodological competency requirements" that pay higher wages to those who possess high levels of these skills. None of these skills, however, could be directly linked to the entrepreneurial skills described before. The REFLEX project includes a wider set of skills, some of them directly linked to the definition proposed by Kirzner (1999), thus permitting a more direct analysis of entrepreneurial skills.

Finally, our research is related to the literature on corporate entrepreneurship (also known as intrapreneurship or entrepreneurial employees). Corporate entrepreneurship refers to the firm's strategy of being closely attentive to entrepreneurial opportunities and involves the different level managers of the firm. Kuratko and Audretsch (2013) provide a review of this literature. They distinguish two domains of corporate entrepreneurship: corporate venturing and strategic entrepreneurship. The former is about creating new businesses within the corporation, while the latter domain attempts to strengthen the competitive advantage of the existing businesses in the 
corporation. Unlike this literature, we do not focus on the effect of entrepreneurial activity within the corporation on its innovation performance; rather, we study the effect of worker's entrepreneurial skills on wages. Our focus is, therefore, on the average returns to entrepreneurial skills in wage employment.

\section{Data and methodology}

\section{Data}

We use the REFLEX data (Research into Employment and professional FLEXibility), a survey of tertiary graduates from the year 2000 that were interviewed five years later. It covers 15 countries and collects information on individual characteristics, first and current job, as well as tertiary-level study program characteristics. Moreover, individuals report measures of 19 individual competencies and their correspondent job requirement level. ${ }^{3}$

We choose Spain in our analysis. Spain has two main advantages over other countries. On the one hand, it has a large sample size. On the other hand, given its institutional and cultural context, parental background is an important determinant of self-employment in this country. This allows us to use parental education as the exclusion restriction on the wage analysis. The response rate of Spain was $22 \%$, which corresponds to 3,912 respondents (Allen and Van der Velden, 2009). ${ }^{4}$ We exclude those individuals above 45 years old and those working 20 or fewer hours weekly. The final sample size is 2,154 individuals, of whom 2,002 are in wage employment (93\%) and 152 are self-employed $(7 \%){ }^{5}$ Table 1 reports the 19 competencies that are included in the survey. Respondents are asked how much each competency is required in their current job and which is their own level of competency. These self-reported measures are evaluated in a 7-level Likert scale (1-very low, 7-very high).

Table 1. Competencies

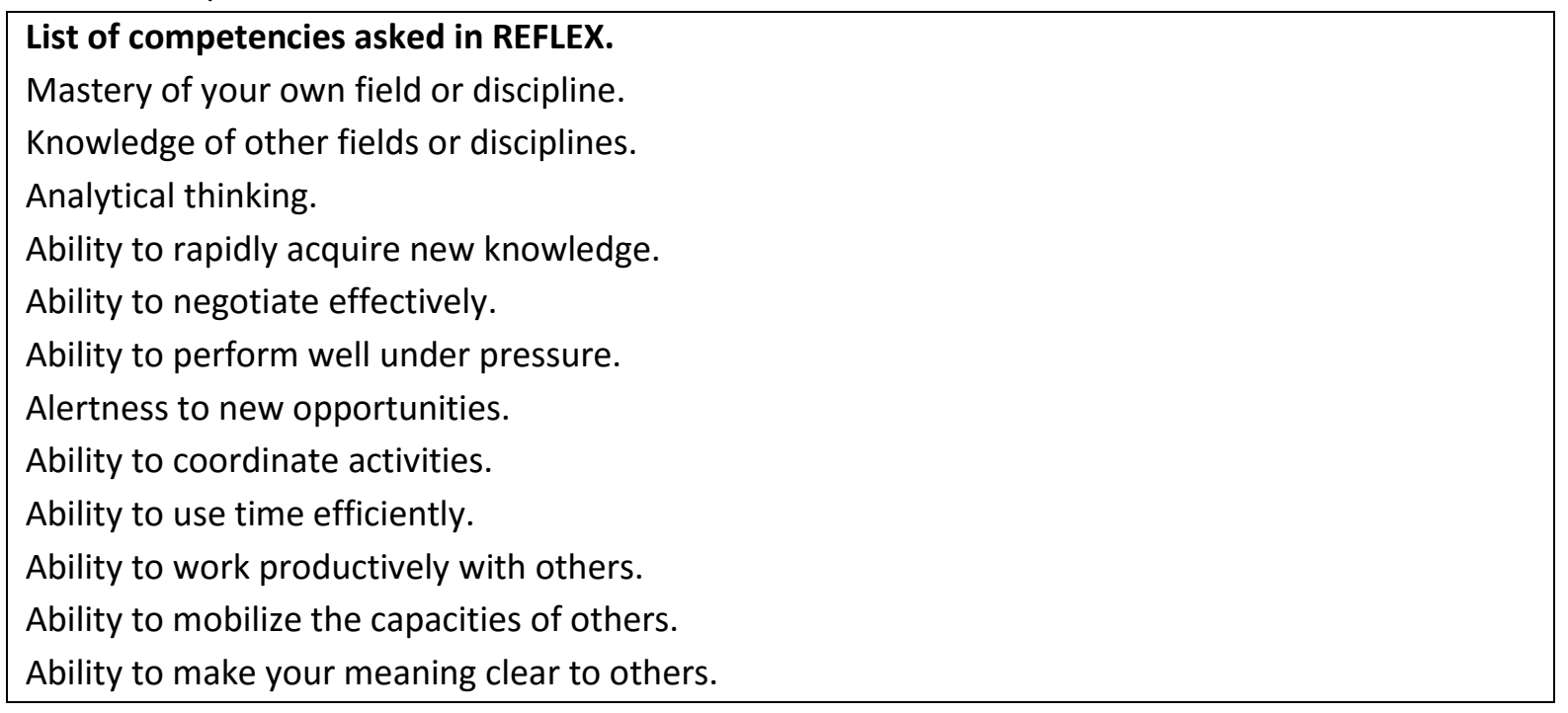

\footnotetext{
${ }^{3}$ A full description of the survey is provided in the report by Allen and Van der Velden (2009). More information is also available in http://www.reflexproject.org.

${ }^{4}$ The response rate in Spain is similar to the one in Belgium and the UK. The average response rate was $31 \%$. This low response rate puts a limitation in our analysis as long as the respondents were not a random sample of the population.

${ }^{5}$ The percentage of self-employed individuals in a comparable sample computed from the EPA survey 2005 is around $8 \%$.
} 
Ability to assert your authority.

Ability to use computers and the internet.

Ability to come up with new ideas and solutions.

Willingness to question your own and others' ideas.

Ability to present products, ideas or reports to an audience.

Ability to write reports, memos or documents.

Ability to write and speak in a foreign language.

We want to disentangle which competencies affect the decision between self-employment and wage employment and whether they are rewarded in wage employment. Similarly, we check whether a wide set of skills matter for self-employment and whether the variety of competencies is rewarded in wage employment. For that, we count how many competencies the individual evaluates at least at level 5 (out of 7). Finally, we investigate whether these competencies increase the income of selfemployed individuals.

The rest of explanatory variables used in the study are gender, age, field of study, grade in secondary education, whether the study program gives access to doctorate studies, and experience in the labor market. Moreover, since the level of competencies is asked five years after graduation and could be endogenous to the current job characteristics, some specifications include additional job controls for those in wage employment. These are the required level of competency in the job, firm size, public/private sector and permanency of the contract. We provide descriptive statistics of all the variables in Table 2.

From Table 2 we observe that there are relatively more women in wage employment than in selfemployment. Moreover, Engineering is the field of study most widely represented within selfemployment, while Social Sciences are more common in wage employment. As for parental education, the average education level of the father is higher in the self-employed sample. Concerning competencies, self-employed individuals report on average a higher level of all of them except for the ability to work with others and the ability to use computers. This is true with respect to both, the own level of competencies and the job requirement level. The largest differences across the two samples with respect to the own level of competency are in alertness to opportunities, ability to work under pressure and ability to negotiate. The largest differences with respect to the required level of competencies occur in alertness to opportunities, ability to negotiate and mastery of own field, by order of importance.

\section{Methodology}

We estimate a Heckman selection model. The system of equations to estimate is the following:

$$
\begin{aligned}
& y_{1}^{*}=x_{1}^{\prime} \beta_{1}+\varepsilon_{1}, \\
& y_{2}^{*}=x_{2}^{\prime} \beta_{2}+\varepsilon_{2},
\end{aligned}
$$

where $y_{1}^{*}$ is the latent variable that determines whether the individual will choose wage employment or self-employment, $y_{2}^{*}$ is the wage in a salaried job, which is only observed when $y_{1}^{*}>0$, and $\varepsilon_{1}$ and $\varepsilon_{2}$ are the error terms, assumed jointly normally distributed and homoscedastic, with $\operatorname{var}\left(\varepsilon_{1}\right)=1$, $\operatorname{var}\left(\varepsilon_{2}\right)=\sigma^{2}$ and $\operatorname{corr}\left(\varepsilon_{1}, \varepsilon_{2}\right)=\rho$.

Individuals choose wage employment when the utility of such a choice is larger than the utility of selfemployment. 


$$
y_{1}^{*}=u(\text { wage employment })-u(\text { self -employment }) .
$$

We do not observe utilities, but whether they decided to be self-employed or in wage employment. The observed variable is then

$$
y_{1}=\left\{\begin{array}{l}
1 \text { if } y_{1}^{*}>0 \\
0 \text { if } y_{1}^{*} \leq 0
\end{array} .\right.
$$

We use father's education as the exclusion restriction. Parental education represents the level of financial and other help that the individual might get from the family to engage in self-employment. Since parental education is likely to influence individual's ability, and therefore his/her wage, we introduce the individual's grade in secondary education as a control. We argue that any other effect of parental education on wage is driven through education, which is also controlled for in the analysis. Therefore, we consider father's education a good exclusion restriction for our analysis. We acknowledge, though, that a more accurate ability measure would be desirable.

The likelihood function for this model is

$$
L=\prod_{i=1}^{n}\left\{\operatorname{Pr}\left(y_{1 i}^{*} \leq 0\right)\right\}^{1-y_{1 i}}\left\{f\left(y_{2 i} \mid y_{1 i}^{*}>0\right) \times \operatorname{Pr}\left(y_{1 i}^{*}>0\right)\right\}^{y_{1 i}} .
$$

We estimate different specifications. In all of them, the explanatory variable of interest is the own level of competency. The results from these estimations allow us to identify correlations. To argue causality we would need to have exogeneity between competencies and employment type as well as between competencies and wages. However, information on competencies, employment type and wages was all asked at the same point in time. The own level of competencies might therefore be influenced by the job characteristics of the individual. Unfortunately, given the data we have, the only choice to lessen this endogeneity problem is to add job characteristics as controls. Therefore, we add the required level of competency and other job characteristics in some specifications.

\section{Results}

As mentioned in the previous section, we estimate a Heckman selection model where the logarithm of gross hourly wage is the dependent variable in the main equation and whether individuals are employed in wage employment is the selection variable.

Table 3 shows the results of the basic specification. In the first 19 estimations we include the own level of one competency in both, main and selection equations. In the last column, we introduce the variable measuring the variety of skills. All equations control for gender, age, field of study, grade in secondary education, whether the study program gives access to doctorate studies and labor market experience. Whether father has tertiary education is the exclusion restriction in the selection equation. Only three competencies have a significantly negative coefficient in the selection equation: alertness to opportunities, ability to work under pressure and knowledge of other fields. These three competencies are therefore associated to a higher likelihood to become self-employed (lower likelihood of wage employment). In contrast, the ability to work with others has a positive and significant coefficient, indicating that this ability tends to increase the probability to choose wage employment. Alertness to opportunities is the competency more directly linked to entrepreneurial skills in the entrepreneurship literature (Valliere, 2013). The two other competencies closer to entrepreneurship - ability to come up with ideas (creativity) and willingness to question them (counterfactual thinking) - do not seem to affect the decision to become self-employed. The variety of skills, which is usually associated to entrepreneurs, is also not significant in our estimation. 
While only a few competencies appear relevant for the decision to become self-employed, many of them are rewarded in wage employment. This is the case for the following competencies: the mastery of own and other fields, analytical thinking, ability to work with others, ability to mobilize others, ability to assert authority, ability to come up with ideas and solutions, willingness to question ideas, ability to present to an audience, and ability to speak a foreign language. This result is in line with the results in Hartog et al. (2010), who observe that clerical and verbal skills are most rewarded in the wage employment. The number of skills is also positively rewarded in wage employment. This positive result has also being found in Bublitz and Noseleit (2014). Other studies find a non-significant effect (Hartog et al., 2010).

It is important to notice that among the competencies that increase the likelihood of self-employment (alertness to opportunities, ability to work under pressure and knowledge of other fields) only knowledge of other fields is rewarded in wage employment. This suggests that the benefits of those policies promoting them come mostly from the increase in the likelihood of self-employment.

Concerning the other variables in the model, we obtain that self-employment attracts rather young individuals, with low grade in secondary education and some experience in the labor market. ${ }^{6}$ Selfemployed individuals graduated mostly from engineering, agriculture and veterinarian studies, health and services. In most cases having a father with tertiary education increases the likelihood of selfemployment as predicted.

Individuals in wage employment earn more if they have studied a long program, especially in education, engineering or health. Women earn on average lower wages than men do, while we find no effect of age on wages. Individuals with a higher grade in secondary education (individuals with higher cognitive ability) earn more on average.

In Table 4 we introduce in the main equation the job requirement level of each competency together with the own level reported. The own level of competencies reported might be strongly influenced by the type of job of the individual. It is therefore important to control for the job requirement level of these competencies. Results on the selection into wage employment do not change. However, the positive effects on wages of all competencies - except analytical thinking - disappear. Moreover, the own level of ability to use time efficiently and write reports show negative returns. Results reveal that wages are determined by the requirement level of each competency.

Table 5 adds some other job characteristics, such as whether it is in the public sector, whether the contract is permanent and the firm size. All these variables increase the earnings of the individual. Results on competencies remain unchanged. None of the competencies that increase the likelihood of self-employment is rewarded in wage employment.

In Table 6, we estimate the Heckman selection model with all the competencies included simultaneously. In column (1) we introduce the own level of competencies, field of study and individual controls. In column (2), we add the required level of competencies to the previous estimation. Column (3) reports the results after adding other job characteristics in the estimation. Analytical thinking, ability to work with others and to assert authority are rewarded in wage employment, even when controlling for job characteristics (column (3)). The competencies that increase the probability of self-employment are alertness to opportunities, ability to mobilize others and knowledge of other fields. ${ }^{7}$ Ability to work under pressure loses significance when all

\footnotetext{
${ }^{6}$ Recall that the dependent variable in the selection equation is whether the individual is in wage employment, so the sign of the coefficients refer to the likelihood to be in wage employment.

${ }^{7}$ The knowledge of other fields may disguise the "Jack-of-all-trades" type of individuals described in Hartog et al. (2010)
} 
competencies are introduced simultaneously. In any case, none of the competencies that increase self-employment is positively rewarded in wage employment. Instead, the ability to mobilize others appears negatively rewarded in wage employment. Even more, the entrepreneurial competencies such as ability to work under pressure and alertness to opportunities reveal a negative coefficient in the required level of competency in the job.

Finally, we estimate the effect of the level of competencies on the earnings of self-employed. Results are, however, to be taken with caution since the sample of self-employed is very small (152 observations). Only the knowledge of other fields and the ability to write reports increase significantly the earnings of self-employed individuals. The knowledge of other fields could be linked to the theory of entrepreneurs with a balanced set of skills being more successful. Self-employed individuals that studied a long program and engineering earn the most. Most of the entrepreneurial skills do not increase income of the self-employed. Therefore, they seem to increase the likelihood of selfemployment, but not its quality. Only knowledge of other fields fosters self-employment and its quality.

\section{Conclusions}

This paper identifies and estimates the effect of entrepreneurial competencies on wages for higher education graduates in Spain. Entrepreneurial skills are understood as those competencies that increase the likelihood of self-employment. We employ a sample of university graduates from the REFLEX survey, who graduated in the year 2000, and were interviewed five years later.

Alertness to new opportunities, ability to mobilize others and knowledge of other fields are the competencies that increase the probability of self-employment in Spain. In contrast, the ability to work with others increases the likelihood to be in wage employment. We find that the competencies that increase self-employment are generally not rewarded in neither wage employment nor selfemployment. They only augment the probability to become self-employed. Therefore, our results suggest that policies promoting entrepreneurial skills of individuals do not have additional benefits outside self-employment. The only exception is knowledge of other fields, which increases both the likelihood of self-employment and its income. It is, however, not rewarded in wage employment.

This work has two main policy implications. On the one hand, we identify which competencies should be included in entrepreneurship education in Spain: alertness to new opportunities, ability to mobilize others and knowledge of other fields. On the other hand, we find that these competencies are not rewarded in wage employment, so the benefits of policies promoting entrepreneurial education remain within self-employment activity only. This should be taken into account when deciding upon policies fostering entrepreneurship. 


\section{References}

ALDRICH, H. E. \& KIM, P. H. 2007. Small worlds, infinite possibilities? How social networks affect entrepreneurial team formation and search. Strategic Entrepreneurship Journal, 1, 147-165.

ALLEN, J. \& VAN DER VELDEN, R. 2009. Competencies and Early Labour Market Careers of Higher Education Graduates. Higher Education as a Generator of Strategic Competencies (HEGESCO). Ljubljana: University of Ljubljana.

ALLEN, J. \& VELDEN, R. V. D. 2001. Educational Mismatches vs. Skill Mismatches: Effects of Wages, Job Satisfaction and On-the-job Search. Oxford Economic Papers, 3, 434-452.

ARDICHVILI, A., CARDOZO, R. \& RAY, S. 2003. A theory of entrepreneurial opportunity identification and development. Journal of Business Venturing, 18, 105-123.

ARENIUS, P. \& CLERCQ, D. D. 2005. A Network-based Approach on Opportunity Recognition. Small Business Economics, 24, 249-265.

ARENIUS, P. \& MINNITI, M. 2005. Perceptual Variables and Nascent Entrepreneurship. Small Business Economics, 24, 233-247.

BAE, T. J., QIAN, S., MIAO, C. \& FIET, J. O. 2014. The Relationship Between Entrepreneurship Education and Entrepreneurial Intentions: A Meta-Analytic Review. Entrepreneurship Theory and Practice, 38, 217-254.

BARON, R. A. 2004. The cognitive perspective: a valuable tool for answering entrepreneurship's basic "why" questions. Journal of Business Venturing, 19, 221-239.

BARON, R. A. \& ENSLEY, M. D. 2006. Opportunity recognition as the detection of meaningful patterns: evidence from comparisons of novice and experienced entrepreneurs. Management Science, $52,1331-1344$.

BÉCHARD, J.-P. \& GRÉGOIRE, D. 2005. Entrepreneurship Education Research Revisited: The Case of Higher Education. Academy of Management Learning \& Education, 4, 22-43.

BECKER, G. 1993. Human Capital. A Theoretical and Empirical Analysis with Special Reference to Education, Chicago and London, The University of Chicago Press.

BIESMA, R. G., PAVLOVA, M., VAN MERODE, G. G. \& GROOT, W. 2007. Using conjoint analysis to estimate employers preferences for key competencies of master level Dutch graduates entering the public health field. Economics of Education Review, 26, 375-386.

BLANCHFLOWER, D. G. \& OSWALD, A. J. 1998. What Makes an Entrepreneur? Journal of Labor Economics, 16, 26-60.

BUBLITZ, E. \& NOSELEIT, F. 2014. The skill balancing act: when does broad expertise pay off? Small Business Economics, 42, 17-32.

CALIENDO, M., FOSSEN, F. \& KRITIKOS, A. 2014. Personality characteristics and the decisions to become and stay self-employed. Small Business Economics, 42, 787-814.

CARDON, M. S., GREGOIRE, D. A., STEVENS, C. E. \& PATEL, P. C. 2013. Measuring entrepreneurial passion: conceptual foundations and scale validation. Journal of Business Venturing, 28, 373396.

CARLSSON, B., ACS, Z. J., AUDRETSCH, D. B. \& BRAUNERHJELM, P. 2009. Knowledge creation, entrepreneurship, and economic growth: a historical review. Industrial and Corporate Change, $18,1193-1229$.

DOMS, M., LEWIS, E. \& ROBB, A. 2010. Local labor force education, new business characteristics, and firm performance. Journal of Urban Economics, 67, 61-77.

ESTRIN, S., KOROSTELEVA, J. \& MICKIEWICZ, T. 2013. Which institutions encourage entrepreneurial growth aspirations? Journal of Business Venturing, 28, 564-580.

EUROPEAN COMMISSION 2009. An EU Strategy for Youth - Investing and Empowering: A renewed open method of coordination to address youth challenges and opportunities. In: COMMUNITIES, C. O. T. E. (ed.) 546. Brussels.

EUROPEAN COMMISSION 2013. Entrepreneurship 2020 Action Plan. Reigniting the entrepreneurial spirit in Europe.: European Commission. 
FIET, J. O. 2001. The theoretical side of teaching entrepreneurship. Journal of Business Venturing, 16, $1-24$.

GAGLIO, C. M. 2004. The role of mental simulations and counterfactual thinking in the opportunity identification process. Entrepreneurship Theory and Practice, 28, 533-552.

GARCIA-ARACIL, A. 2009. European graduates' level of satisfaction with higher education. Higher Education, 57, 1-21.

GARCIA-ARACIL, A. \& VAN DER VELDEN, R. 2008. Competencies for young European higher education graduates: labor market mismatches and their payoffs. Higher Education, 55, 219-239.

GARCIA-ARACIL, A., MORA, J. G. \& VILA, L. E. 2004. The rewards of human capital competences for young European higher education graduates. Tertiary Education and Management, 10, 287305.

GRANOVETTER, M. S. 1973. The Strength of Weak Ties. The American Journal of Sociology, 78, 13601380.

GROSSMANN, V. 2009. Entrepreneurial innovation and economic growth. Journal of Macroeconomics, 31, 602-613.

HAASE, H. \& LAUTENSCHLÄGER, A. 2011. The 'Teachability Dilemma' of entrepreneurship. International Entrepreneurship and Management Journal, 7, 145-162.

HAMILTON, B. H. 2000. Does Entrepreneurship Pay? An Empirical Analysis of the Returns to SelfEmployment. Journal of Political Economy, 108, 604-631.

HANUSHEK, E. A. \& ZHANG, L. 2009. Quality Consistent Estimates of International Schooling and Skill Gradients. Journal of Human Capital, 3, 107-143.

HARTOG, J., VAN PRAAG, M. \& VAN DER SLUIS, J. 2010. If you are so smart, why aren't you an entrepreneur? Returns to cognitive and social ability: entrepreneurs versus employees. Journal of Economics \& Management Strategy, 19, 947-989.

HECKMAN, J. J. \& KAUTZ, T. 2012. Hard evidence on soft skills. Labour Economics, 19, 451-464.

HECKMAN, J. J., LOCHNER, L. J. \& TODD, P. E. 2003. Fifty Years of Mincer Earnings Regressions. NBER Working Papers, 9732.

HEIJKE, H., MENG, C. \& RAMAEKERS, G. 2003. An investigation into the role of human capital competences and their pay-off. International Journal of Manpower, 24, 750-773.

HYYTINEN, A., ILMAKUNNAS, P. \& TOIVANEN, O. 2013. The return-to-entrepreneurship puzzle. Labour Economics, 20, 57-67.

IYIGUN, M. F. \& OWEN, A. L. 1999. Entrepreneurs, Professionals, and Growth. Journal of Economic Growth, 4, 213-232.

KIRZNER, I. 1999. Creativity and/or Alertness: A Reconsideration of the Schumpeterian Entrepreneur. The Review of Austrian Economics, 11, 5-17.

KIRZNER, I. M. 1973. Competition and entrepreneurship, Chicago, Chicago University Press.

KURATKO, D. F. 2005. The Emergence of Entrepreneurship Education: Development, Trends, and Challenges. Entrepreneurship Theory and Practice, 29, 577-598.

KURATKO, D. F. \& AUDRETSCH, D. B. 2013. Clarifying the domains of corporate entrepreneurship. International Entrepreneurship and Management Journal, 9, 323-335.

LAZEAR, E. P. 2004. Balanced Skills and Entrepreneurship. The American Economic Review, 94, 208211.

LAZEAR, EDWARD P. 2005. Entrepreneurship. Journal of Labor Economics, 23, 649-680.

MARTIARENA, A. 2013. What's so entrepreneurial about intrapreneurs? Small Business Economics, 40, 27-39.

MARTIN, B. C., MCNALLY, J. J. \& KAY, M. J. 2013. Examining the formation of human capital in entrepreneurship: A meta-analysis of entrepreneurship education outcomes. Journal of Business Venturing, 28, 211-224.

MARTíNEZ, D., MORA, J.-G. \& VILA, L. E. 2007. Entrepreneurs, the Self-employed and Employees amongst Young European Higher Education Graduates. European Journal of Education, 42, 99117.

MINCER, J. A. 1974. Schooling, Experience, and Earnings, New York, Columbia University Press. 
MORA, J.-G., GARCIA-ARACIL, A., CAROT, J. M. \& VILA, L. E. 2006. Monetary rewards and competences of young european graduates. Higher Education Management and Policy, 18, 29-44.

O'CONNOR, A. 2013. A conceptual framework for entrepreneurship education policy: Meeting government and economic purposes. Journal of Business Venturing, 28, 546-563.

OBERSCHACHTSIEK, D. 2012. The experience of the founder and self-employment duration: a comparative advantage approach. Small Business Economics, 39, 1-17.

OOSTERBEEK, H., VAN PRAAG, M. \& IJSSELSTEIN, A. 2010. The impact of entrepreneurship education on entrepreneurship skills and motivation. European Economic Review, 54, 442-454.

PINCHOT, G. 1985. Intrapreneuring., New York: Harper and Row.

POSCHKE, M. 2013. Who becomes an entrepreneur? Labor market prospects and occupational choice. Journal of Economic Dynamics and Control, 37, 693-710.

PRAAG, C. M. \& VERSLOOT, P. 2007. What is the value of entrepreneurship? A review of recent research. Small Business Economics, 29, 351-382.

SHANE, S., LOCKE, E. A. \& COLLINS, C. J. 2003. Entrepreneurial motivation. Human Resource Management Review, 13, 257-279.

SHARMA, P. \& CHRISMAN, J. J. 1999. Toward a reconciliation of the definitional issues in the field of corporate entrepreneurship. Entrepreneurship Theory and Practice, 23, 11-27.

STUETZER, M., OBSCHONKA, M., DAVIDSSON, P. \& SCHMITT-RODERMUND, E. 2013. Where do entrepreneurial skills come from? Applied Economics Letters, 20, 1183-1186.

STUETZER, M., OBSCHONKA, M. \& SCHMITT-RODERMUND, E. 2012. Balanced skills among nascent entrepreneurs. Small Business Economics, 41, 93-114.

TANG, J., KACMAR, K. M. \& BUSENITZ, L. 2012. Entrepreneurial alertness in the pursuit of new opportunities. Journal of Business Venturing, 27, 77-94.

TEIJEIRO, M., RUNGO, P. \& FREIRE, M. J. 2013. Graduate competencies and employability: The impact of matching firms' needs and personal attainments. Economics of Education Review.

VALLIERE, D. 2013. Towards a schematic theory of entrepreneurial alertness. Journal of Business Venturing, 28, 430-442.

VAN DER SLUIS, J. \& VAN PRAAG, M. 2004. Economic returns to education for entrepreneurs: The development of a neglected child in the family of economics of education? Sweedish Economic Policy Review, 11, 183-226.

VAN DER SLUIS, J., VAN PRAAG, M. \& VIJVERBERG, W. 2008. EDUCATION AND ENTREPRENEURSHIP SELECTION AND PERFORMANCE: A REVIEW OF THE EMPIRICAL LITERATURE. Journal of Economic Surveys, 22, 795-841.

VON GRAEVENITZ, G., HARHOFF, D. \& WEBER, R. 2010. The effects of entrepreneurship education. Journal of Economic Behavior \& Organization, 76, 90-112. 
Table 2. Descriptive statistics.

\begin{tabular}{lcccc} 
& \multicolumn{2}{c}{ Self-employed } & \multicolumn{2}{c}{ Wage employment } \\
variable & mean & sd & mean & sd \\
\hline Female & 0,546 & 0,499 & 0,632 & 0,482 \\
Long program* & 0,730 & 0,445 & 0,693 & 0,461 \\
Grade secondary education & 2,920 & 0,953 & 2,900 & 0,943 \\
Father w/ tertiary education & 0,336 & 0,474 & 0,246 & 0,431 \\
log(hourly wage) & 2,191 & 0,371 & 2,154 & 0,377 \\
Public sector &. &. & 0,322 & 0,467 \\
Permanent contract &. &. & 0,616 & 0,486 \\
Medium firm size &. &. & 0,193 & 0,395 \\
Large firm &. &. & 0,530 & 0,499 \\
Months of experience &. &. & 50,41 & 15,30 \\
Field of study & & & & \\
Education & 0,079 & 0,271 & 0,108 & 0,311 \\
Humanities and arts & 0,092 & 0,290 & 0,064 & 0,245 \\
Social Sciences & 0,211 & 0,409 & 0,349 & 0,477 \\
Science \& Maths & 0,086 & 0,281 & 0,148 & 0,355 \\
Engineering & 0,303 & 0,461 & 0,154 & 0,361 \\
Agriculture \& Vet & 0,066 & 0,249 & 0,041 & 0,198 \\
Health and welfare & 0,145 & 0,353 & 0,130 & 0,337 \\
Services & 0,020 & 0,140 & 0,006 & 0,078 \\
Observations & 152 & & 2002 &
\end{tabular}

Competencies (Own Level):

\section{Competency}

Mastery of own field

Knowledge other fields

Analytical thinking

Ability to learn

Ability to negotiate

Ability to work under pressure

Alertness to opportunities

Ability to coordinate

Ability to use time efficiently

Ability to work with others

Ability to mobilize others

Ability to make meaning clear

Ability to assert authority

Ability to use computers

Ability to come up with ideas

Willingness to question ideas

Ability to present

Ability to write

Foreign language

Number of skills
Self-employed Wage employment Self-employed Wage employment

$\begin{array}{cccccccc}\text { mean } & \text { sd } & \text { mean } & \text { sd } & \text { mean } & \text { sd } & \text { mean } & \text { sd } \\ 5,276 & 0,978 & 5,187 & 1,070 & 5,632 & 1,300 & 5,161 & 1,481 \\ 4,553 & 1,155 & 4,349 & 1,225 & 4,388 & 1,536 & 4,125 & 1,535 \\ 5,171 & 1,233 & 5,067 & 1,219 & 5,086 & 1,451 & 4,764 & 1,523 \\ 5,789 & 0,960 & 5,725 & 1,030 & 5,388 & 1,352 & 5,226 & 1,420 \\ 4,757 & 1,223 & 4,501 & 1,472 & 5,079 & 1,713 & 4,515 & 1,857 \\ 5,717 & 1,124 & 5,419 & 1,273 & 5,704 & 1,249 & 5,374 & 1,530 \\ 5,086 & 1,212 & 4,687 & 1,336 & 4,908 & 1,498 & 4,347 & 1,738 \\ 5,546 & 1,173 & 5,388 & 1,212 & 5,493 & 1,424 & 5,204 & 1,524 \\ 5,572 & 1,171 & 5,494 & 1,161 & 5,803 & 1,191 & 5,555 & 1,350 \\ 5,533 & 1,286 & 5,815 & 1,167 & 5,316 & 1,633 & 5,477 & 1,558 \\ 5,000 & 1,287 & 4,759 & 1,321 & 4,921 & 1,609 & 4,713 & 1,704 \\ 5,539 & 1,167 & 5,486 & 1,121 & 5,763 & 1,126 & 5,618 & 1,332 \\ 4,895 & 1,246 & 4,712 & 1,377 & 5,178 & 1,505 & 4,763 & 1,730 \\ 5,533 & 1,207 & 5,645 & 1,231 & 5,138 & 1,456 & 5,261 & 1,507 \\ 5,474 & 1,029 & 5,295 & 1,138 & 5,513 & 1,229 & 5,169 & 1,463 \\ 5,309 & 1,246 & 5,297 & 1,206 & 5,099 & 1,436 & 4,722 & 1,562 \\ 4,803 & 1,478 & 4,745 & 1,536 & 4,875 & 1,791 & 4,624 & 1,829 \\ 5,474 & 1,145 & 5,425 & 1,257 & 5,454 & 1,491 & 5,086 & 1,666 \\ 3,941 & 1,784 & 3,839 & 1,841 & 3,349 & 1,930 & 3,368 & 2,070 \\ 14,092 & 3,897 & 13,387 & 4,263 & 13,079 & 4,524 & 12,040 & 5,016\end{array}$

* dummy indicating whether studies provide direct access to doctorate. 
Table 3a. Heckman selection model, benchmark model.

\begin{tabular}{|c|c|c|c|c|c|c|c|c|c|c|}
\hline & \multicolumn{2}{|c|}{ Mastery of own field } & \multicolumn{2}{|c|}{ Knowledge other fields } & \multicolumn{2}{|c|}{ Analytical thinking } & \multicolumn{2}{|c|}{ Ability to learn } & \multicolumn{2}{|c|}{ Ability to negotiate } \\
\hline & Ln(wage) & Selection & Ln(wage) & Selection & Ln(wage) & Selection & Ln(wage) & Selection & Ln(wage) & Selection \\
\hline \multirow[t]{2}{*}{ Own level of competence } & $0.013^{*}$ & -0.013 & $0.019 * * *$ & $-0.067 * *$ & $0.041 * * *$ & -0.005 & 0.011 & -0.040 & 0.008 & -0.043 \\
\hline & $(0.008)$ & $(0.036)$ & $(0.007)$ & $(0.033)$ & $(0.007)$ & $(0.032)$ & $(0.008)$ & $(0.042)$ & $(0.006)$ & $(0.028)$ \\
\hline \multirow[t]{2}{*}{ Female } & $-0.094 * * *$ & 0.014 & $-0.101 * * *$ & 0.041 & $-0.085 * * *$ & 0.014 & $-0.104 * * *$ & 0.053 & $-0.094 * * *$ & 0.014 \\
\hline & $(0.019)$ & $(0.084)$ & $(0.019)$ & $(0.086)$ & $(0.019)$ & $(0.084)$ & $(0.019)$ & $(0.086)$ & $(0.019)$ & $(0.084)$ \\
\hline \multirow[t]{2}{*}{ Age } & -0.001 & $-0.031 * *$ & $0.006^{*}$ & -0.024 & -0.000 & $-0.036 * *$ & $0.006^{*}$ & -0.026 & -0.001 & $-0.032 * *$ \\
\hline & $(0.004)$ & $(0.016)$ & $(0.004)$ & $(0.016)$ & $(0.004)$ & $(0.015)$ & $(0.004)$ & $(0.016)$ & $(0.004)$ & $(0.016)$ \\
\hline \multirow[t]{2}{*}{ Long program } & $0.091 * * *$ & -0.136 & $0.120 * * *$ & $-0.175^{*}$ & $0.075 * * *$ & -0.117 & $0.120 * * *$ & -0.169 & $0.089 * * *$ & -0.137 \\
\hline & $(0.022)$ & $(0.097)$ & $(0.021)$ & $(0.103)$ & $(0.022)$ & $(0.098)$ & $(0.021)$ & $(0.103)$ & $(0.022)$ & $(0.097)$ \\
\hline \multirow[t]{2}{*}{ Grade secondary education } & $0.056 * * *$ & $0.099 * *$ & $0.057 * * *$ & 0.015 & $0.052 * * *$ & $0.102 * *$ & $0.056 * * *$ & 0.021 & $0.057 * * *$ & $0.089 * *$ \\
\hline & $(0.010)$ & $(0.044)$ & $(0.010)$ & $(0.046)$ & $(0.010)$ & $(0.044)$ & $(0.010)$ & $(0.046)$ & $(0.010)$ & $(0.045)$ \\
\hline \multirow[t]{2}{*}{ Experience } & $0.003 * * *$ & $-0.008 * * *$ & $0.004 * * *$ & $-0.012 * * *$ & $0.003 * * *$ & $-0.009 * * *$ & $0.004 * * *$ & $-0.012 * * *$ & $0.003 * * *$ & $-0.008 * * *$ \\
\hline & $(0.001)$ & $(0.003)$ & $(0.001)$ & $(0.003)$ & $(0.001)$ & $(0.003)$ & $(0.001)$ & $(0.003)$ & $(0.001)$ & $(0.003)$ \\
\hline \multicolumn{11}{|l|}{ Fields of study } \\
\hline \multirow[t]{2}{*}{ Education } & $0.128 * * *$ & 0.034 & $0.172 * * *$ & -0.266 & $0.130 * * *$ & 0.024 & $0.178 * * *$ & $-0.276^{*}$ & $0.127 * * *$ & 0.034 \\
\hline & $(0.032)$ & $(0.156)$ & $(0.032)$ & $(0.162)$ & $(0.032)$ & $(0.154)$ & $(0.032)$ & $(0.162)$ & $(0.032)$ & $(0.155)$ \\
\hline \multirow[t]{2}{*}{ Humanities } & -0.040 & -0.218 & 0.036 & -0.245 & -0.039 & -0.193 & 0.039 & -0.258 & -0.034 & -0.235 \\
\hline & $(0.037)$ & $(0.157)$ & $(0.037)$ & $(0.165)$ & $(0.037)$ & $(0.158)$ & $(0.037)$ & $(0.165)$ & $(0.037)$ & $(0.157)$ \\
\hline \multirow[t]{2}{*}{ Science \&Maths } & 0.044 & 0.099 & 0.029 & 0.038 & 0.028 & 0.089 & 0.029 & 0.043 & $0.047 *$ & 0.075 \\
\hline & $(0.027)$ & $(0.142)$ & $(0.027)$ & $(0.144)$ & $(0.027)$ & $(0.140)$ & $(0.027)$ & $(0.144)$ & $(0.027)$ & $(0.143)$ \\
\hline \multirow[t]{2}{*}{ Engineering } & $0.132 * * *$ & $-0.536 * * *$ & $0.207 * * *$ & $-0.582 * * *$ & $0.116^{* * * *}$ & $-0.510 * * *$ & $0.208 * * *$ & $-0.576 * * *$ & $0.130 * * *$ & $-0.544 * * *$ \\
\hline & $(0.028)$ & $(0.119)$ & $(0.028)$ & $(0.123)$ & $(0.028)$ & $(0.119)$ & $(0.028)$ & $(0.123)$ & $(0.028)$ & $(0.118)$ \\
\hline \multirow[t]{2}{*}{ Agriculture\&Vet } & 0.021 & $-0.439 * *$ & $0.088 * *$ & $-0.493 * * *$ & 0.014 & $-0.411 * *$ & $0.091 * *$ & $-0.506 * * *$ & 0.021 & $-0.443 * *$ \\
\hline & $(0.044)$ & $(0.188)$ & $(0.044)$ & $(0.190)$ & $(0.044)$ & $(0.186)$ & $(0.044)$ & $(0.190)$ & $(0.044)$ & $(0.189)$ \\
\hline \multirow[t]{2}{*}{ Health } & $0.070 * *$ & $-0.235 *$ & $0.140 * * *$ & $-0.397 * * *$ & $0.066^{* *}$ & $-0.225^{*}$ & $0.140 * * *$ & $-0.387 * * *$ & $0.073 * *$ & $-0.235^{*}$ \\
\hline & $(0.030)$ & $(0.136)$ & $(0.029)$ & $(0.139)$ & $(0.029)$ & $(0.136)$ & $(0.030)$ & $(0.138)$ & $(0.030)$ & $(0.138)$ \\
\hline \multirow[t]{2}{*}{ Services } & 0.102 & $-0.643 *$ & $0.282 * * *$ & $-0.619 *$ & 0.088 & -0.587 & $0.282 * * *$ & $-0.628 *$ & 0.099 & $-0.613 *$ \\
\hline & $(0.107)$ & $(0.365)$ & $(0.107)$ & $(0.376)$ & $(0.107)$ & $(0.365)$ & $(0.108)$ & $(0.374)$ & $(0.107)$ & $(0.371)$ \\
\hline \multirow[t]{2}{*}{ Father w/ tertiary educ. } & & $-0.220 * * *$ & & -0.044 & & $-0.215^{* * *}$ & & -0.051 & & $-0.216 * * *$ \\
\hline & & $(0.079)$ & & $(0.081)$ & & $(0.078)$ & & $(0.081)$ & & $(0.079)$ \\
\hline \multirow[t]{2}{*}{ Constant } & $1.706^{* * *}$ & $2.944 * * *$ & $1.458 * * *$ & $3.469 * * *$ & $1.568 * * *$ & $3.028 * * *$ & $1.470 * * *$ & $3.409 * * *$ & $1.727 * * *$ & $3.147 * * *$ \\
\hline & $(0.119)$ & $(0.548)$ & $(0.117)$ & $(0.545)$ & $(0.118)$ & $(0.546)$ & $(0.125)$ & $(0.589)$ & $(0.117)$ & $(0.543)$ \\
\hline Observations & 2154 & & 2154 & & 2154 & & 2154 & & 2154 & \\
\hline Censored Obs. & 152 & & 152 & & 152 & & 152 & & 152 & \\
\hline Sigma & .389 & & .386 & & .387 & & .387 & & .389 & \\
\hline Rho & .889 & & -.85 & & .897 & & -.848 & & .886 & \\
\hline Lambda & .346 & & -.328 & & .347 & & -.328 & & .345 & \\
\hline
\end{tabular}


Table 3b. Heckman selection model, benchmark model.

\begin{tabular}{|c|c|c|c|c|c|c|c|c|c|c|}
\hline & \multicolumn{2}{|c|}{ Ability to work under pressure } & \multicolumn{2}{|c|}{ Alertness to opportunities } & \multicolumn{2}{|c|}{ Ability to coordinate } & \multicolumn{2}{|c|}{ Ability to use time efficiently } & \multicolumn{2}{|c|}{ Ability to work with others } \\
\hline & Ln(wage) & Selection & Ln(wage) & Selection & Ln(wage) & Selection & Ln(wage) & Selection & Ln(wage) & Selection \\
\hline \multirow[t]{2}{*}{ Own level of competence } & 0.003 & $-0.063 * *$ & 0.004 & $-0.107 * * *$ & 0.011 & -0.042 & -0.010 & -0.038 & $0.018^{* *}$ & $0.074 * *$ \\
\hline & $(0.007)$ & $(0.032)$ & $(0.006)$ & $(0.031)$ & $(0.007)$ & $(0.032)$ & $(0.007)$ & $(0.033)$ & $(0.007)$ & $(0.029)$ \\
\hline \multirow[t]{2}{*}{ Female } & $-0.095 * * *$ & 0.012 & $-0.095 * * *$ & 0.011 & $-0.097 * * *$ & 0.028 & $-0.091 * * *$ & 0.023 & $-0.098 * * *$ & 0.000 \\
\hline & $(0.019)$ & $(0.085)$ & $(0.019)$ & $(0.083)$ & $(0.019)$ & $(0.084)$ & $(0.019)$ & $(0.085)$ & $(0.019)$ & $(0.085)$ \\
\hline \multirow[t]{2}{*}{ Age } & -0.001 & $-0.033 * *$ & -0.001 & $-0.030 *$ & -0.001 & $-0.035^{* *}$ & -0.002 & $-0.033 * *$ & -0.001 & $-0.029 *$ \\
\hline & $(0.004)$ & $(0.016)$ & $(0.004)$ & $(0.016)$ & $(0.004)$ & $(0.016)$ & $(0.004)$ & $(0.016)$ & $(0.004)$ & $(0.016)$ \\
\hline \multirow[t]{2}{*}{ Long program } & $0.089 * * *$ & -0.123 & $0.087 * * *$ & -0.092 & $0.089 * * *$ & -0.124 & $0.089 * * *$ & -0.135 & $0.091 * * *$ & -0.125 \\
\hline & $(0.022)$ & $(0.098)$ & $(0.022)$ & $(0.097)$ & $(0.022)$ & $(0.097)$ & $(0.022)$ & $(0.097)$ & $(0.022)$ & $(0.097)$ \\
\hline \multirow[t]{2}{*}{ Grade in secondary edu. } & $0.057 * * *$ & $0.098 * *$ & $0.057 * * *$ & $0.091 * *$ & $0.057 * * *$ & $0.098 * *$ & $0.057 * * *$ & $0.101 * *$ & $0.057 * * *$ & $0.102 * *$ \\
\hline & $(0.010)$ & $(0.045)$ & $(0.010)$ & $(0.043)$ & $(0.010)$ & $(0.044)$ & $(0.010)$ & $(0.044)$ & $(0.010)$ & $(0.045)$ \\
\hline \multirow[t]{2}{*}{ Experience } & $0.003 * * *$ & $-0.008 * * *$ & $0.003 * * *$ & $-0.008 * * *$ & $0.003 * * *$ & $-0.008 * * *$ & $0.003 * * *$ & $-0.008 * * *$ & $0.003 * * *$ & $-0.008 * * *$ \\
\hline & $(0.001)$ & $(0.003)$ & $(0.001)$ & $(0.003)$ & $(0.001)$ & $(0.003)$ & $(0.001)$ & $(0.003)$ & $(0.001)$ & $(0.003)$ \\
\hline \multicolumn{11}{|l|}{ Fields of study } \\
\hline \multirow[t]{2}{*}{ Education } & $0.130 * * *$ & 0.030 & $0.127 * * *$ & 0.081 & $0.127 * * *$ & 0.035 & $0.129 * * *$ & 0.041 & $0.124 * * *$ & 0.031 \\
\hline & $(0.032)$ & $(0.157)$ & $(0.032)$ & $(0.153)$ & $(0.032)$ & $(0.155)$ & $(0.032)$ & $(0.156)$ & $(0.032)$ & $(0.157)$ \\
\hline \multirow[t]{2}{*}{ Humanities } & -0.035 & -0.195 & -0.038 & -0.188 & -0.036 & -0.216 & -0.033 & -0.214 & -0.038 & -0.230 \\
\hline & $(0.037)$ & $(0.159)$ & $(0.037)$ & $(0.154)$ & $(0.037)$ & $(0.157)$ & $(0.037)$ & $(0.157)$ & $(0.037)$ & $(0.158)$ \\
\hline \multirow[t]{2}{*}{ Science\&Maths } & 0.044 & 0.096 & $0.045^{*}$ & 0.103 & $0.045^{*}$ & 0.091 & 0.043 & 0.094 & 0.041 & 0.084 \\
\hline & $(0.027)$ & $(0.142)$ & $(0.027)$ & $(0.141)$ & $(0.027)$ & $(0.141)$ & $(0.027)$ & $(0.142)$ & $(0.027)$ & $(0.143)$ \\
\hline \multirow[t]{2}{*}{ Engineering } & $0.130 * * *$ & $-0.535 * * *$ & $0.127 * * *$ & $-0.505^{* * *}$ & $0.128 * * *$ & $-0.533 * * *$ & $0.129 * * *$ & $-0.541 * * *$ & $0.128 * * *$ & $-0.554 * * *$ \\
\hline & $(0.028)$ & $(0.119)$ & $(0.028)$ & $(0.117)$ & $(0.028)$ & $(0.118)$ & $(0.028)$ & $(0.118)$ & $(0.028)$ & $(0.119)$ \\
\hline \multirow[t]{2}{*}{ Agriculture \&Vet } & 0.021 & $-0.440 * *$ & 0.019 & $-0.388 * *$ & 0.019 & $-0.440 * *$ & 0.019 & $-0.453 * *$ & 0.021 & $-0.478 * *$ \\
\hline & $(0.044)$ & $(0.189)$ & $(0.045)$ & $(0.189)$ & $(0.044)$ & $(0.187)$ & $(0.045)$ & $(0.187)$ & $(0.044)$ & $(0.187)$ \\
\hline \multirow[t]{2}{*}{ Health } & $0.073 * *$ & $-0.235^{*}$ & $0.070 * *$ & -0.180 & $0.074 * *$ & $-0.230 *$ & $0.073 * *$ & $-0.230 *$ & $0.067 * *$ & $-0.264 *$ \\
\hline & $(0.030)$ & $(0.138)$ & $(0.030)$ & $(0.134)$ & $(0.030)$ & $(0.137)$ & $(0.030)$ & $(0.136)$ & $(0.030)$ & $(0.137)$ \\
\hline \multirow[t]{2}{*}{ Services } & 0.103 & $-0.663 *$ & 0.096 & $-0.618 *$ & 0.102 & $-0.606^{*}$ & 0.102 & $-0.622 *$ & 0.100 & $-0.649 *$ \\
\hline & $(0.107)$ & $(0.368)$ & $(0.107)$ & $(0.361)$ & $(0.107)$ & $(0.367)$ & $(0.107)$ & $(0.365)$ & $(0.107)$ & $(0.365)$ \\
\hline \multirow[t]{2}{*}{ Father w/ tertiary educ. } & & $-0.210 * * *$ & & $-0.230 * * *$ & & $-0.216 * * *$ & & $-0.213 * * *$ & & $-0.214 * * *$ \\
\hline & & $(0.079)$ & & $(0.076)$ & & $(0.078)$ & & $(0.079)$ & & $(0.080)$ \\
\hline \multirow[t]{2}{*}{ Constant } & $1.744 * * *$ & $3.254 * * *$ & $1.749 * * *$ & $3.318 * * *$ & $1.698 * * *$ & $3.185 * * *$ & $1.822 * * *$ & $3.118 * * *$ & $1.654 * * *$ & $2.402 * * *$ \\
\hline & $(0.121)$ & $(0.562)$ & $(0.117)$ & $(0.534)$ & $(0.120)$ & $(0.560)$ & $(0.122)$ & $(0.567)$ & $(0.122)$ & $(0.558)$ \\
\hline Observations & 2154 & & 2154 & & 2154 & & 2154 & & 2154 & \\
\hline Censored Obs. & 152 & & 152 & & 152 & & 152 & & 152 & \\
\hline Sigma & .388 & & .391 & & .389 & & .39 & & .389 & \\
\hline Rho & .881 & & .908 & & .889 & & .889 & & .888 & \\
\hline Lambda & .342 & & .355 & & .346 & & .346 & & .346 & \\
\hline
\end{tabular}


Table 3c. Heckman selection model, benchmark model.

\begin{tabular}{|c|c|c|c|c|c|c|c|c|c|c|}
\hline & \multicolumn{2}{|c|}{$\begin{array}{l}\text { Ability to mobilize } \\
\text { others }\end{array}$} & \multicolumn{2}{|c|}{$\begin{array}{l}\text { Ability to make meaning } \\
\text { clear }\end{array}$} & \multicolumn{2}{|c|}{$\begin{array}{l}\text { Ability to assert } \\
\text { authority }\end{array}$} & \multicolumn{2}{|c|}{$\begin{array}{l}\text { Ability to use } \\
\text { computers }\end{array}$} & \multicolumn{2}{|c|}{$\begin{array}{c}\text { Ability to come up with } \\
\text { ideas }\end{array}$} \\
\hline & Ln(wage) & Selection & Ln(wage) & Selection & Ln(wage) & Selection & Ln(wage) & Selection & Ln(wage) & Selection \\
\hline \multirow{4}{*}{$\begin{array}{l}\text { Own level of } \\
\text { competence } \\
\text { Female }\end{array}$} & $0.011^{*}$ & -0.028 & 0.012 & 0.009 & $0.027 * * *$ & -0.006 & -0.001 & 0.007 & $0.015^{*}$ & -0.033 \\
\hline & $(0.006)$ & $(0.029)$ & $(0.008)$ & $(0.034)$ & $(0.006)$ & $(0.029)$ & $(0.007)$ & $(0.033)$ & $(0.008)$ & $(0.035)$ \\
\hline & $-0.094 * * *$ & 0.012 & $-0.095 * * *$ & 0.012 & $-0.093 * * *$ & 0.017 & $-0.094 * * *$ & 0.013 & $-0.094 * * *$ & 0.014 \\
\hline & $(0.019)$ & $(0.084)$ & (0.019) & $(0.084)$ & (0.019) & $(0.084)$ & $(0.019)$ & $(0.085)$ & $(0.019)$ & $(0.085)$ \\
\hline \multirow[t]{2}{*}{ Age } & -0.001 & $-0.033 * *$ & -0.001 & $-0.032 * *$ & -0.002 & $-0.035 * *$ & -0.001 & $-0.031 * *$ & -0.001 & $-0.035 * *$ \\
\hline & $(0.004)$ & $(0.016)$ & $(0.004)$ & $(0.016)$ & $(0.004)$ & $(0.016)$ & $(0.004)$ & $(0.016)$ & $(0.004)$ & $(0.016)$ \\
\hline \multirow[t]{2}{*}{ Long program } & $0.088 * * *$ & -0.129 & $0.089 * * *$ & -0.131 & $0.089 * * *$ & -0.124 & $0.089 * * *$ & -0.134 & $0.089 * * *$ & -0.133 \\
\hline & $(0.022)$ & $(0.097)$ & $(0.022)$ & $(0.097)$ & $(0.021)$ & $(0.097)$ & $(0.022)$ & $(0.097)$ & $(0.021)$ & $(0.097)$ \\
\hline \multirow[t]{2}{*}{ Grade in secondary edu. } & $0.057 * * *$ & $0.099 * *$ & $0.056 * * *$ & $0.099 * *$ & $0.057 * * *$ & $0.093 * *$ & $0.057 * * *$ & $0.100 * *$ & $0.057 * * *$ & $0.096 * *$ \\
\hline & $(0.010)$ & $(0.045)$ & $(0.010)$ & $(0.045)$ & $(0.010)$ & $(0.045)$ & $(0.010)$ & $(0.045)$ & $(0.010)$ & $(0.045)$ \\
\hline \multirow[t]{2}{*}{ Experience } & $0.003 * * *$ & $-0.008 * * *$ & $0.003 * * *$ & $-0.008 * * *$ & $0.003 * * *$ & $-0.009 * * *$ & $0.003 * * *$ & $-0.008 * * *$ & $0.003 * * *$ & $-0.008 * * *$ \\
\hline & $(0.001)$ & $(0.003)$ & $(0.001)$ & $(0.003)$ & $(0.001)$ & $(0.003)$ & $(0.001)$ & $(0.003)$ & $(0.001)$ & $(0.003)$ \\
\hline \multicolumn{11}{|l|}{ Field of study: } \\
\hline \multirow[t]{2}{*}{ Education } & $0.128 * * *$ & 0.047 & $0.126 * * *$ & 0.035 & $0.118 * * *$ & 0.025 & $0.128 * * *$ & 0.048 & $0.125^{* * *}$ & 0.032 \\
\hline & $(0.032)$ & $(0.157)$ & $(0.032)$ & $(0.156)$ & $(0.032)$ & $(0.157)$ & $(0.032)$ & $(0.157)$ & $(0.032)$ & $(0.156)$ \\
\hline \multirow[t]{2}{*}{ Humanities } & -0.037 & -0.212 & -0.038 & -0.230 & -0.039 & -0.245 & -0.035 & -0.226 & -0.040 & -0.215 \\
\hline & $(0.037)$ & $(0.157)$ & $(0.037)$ & $(0.157)$ & $(0.037)$ & $(0.157)$ & $(0.037)$ & $(0.157)$ & $(0.037)$ & $(0.158)$ \\
\hline \multirow[t]{2}{*}{ Science\&Maths } & $0.045^{*}$ & 0.093 & 0.044 & 0.096 & $0.044^{*}$ & 0.086 & 0.044 & 0.099 & 0.040 & 0.099 \\
\hline & $(0.027)$ & $(0.142)$ & $(0.027)$ & $(0.142)$ & $(0.027)$ & $(0.142)$ & $(0.027)$ & $(0.142)$ & $(0.027)$ & $(0.142)$ \\
\hline \multirow[t]{2}{*}{ Engineering } & $0.128 * * *$ & $-0.526 * * *$ & $0.131 * * *$ & $-0.535 * * *$ & $0.128 * * *$ & $-0.528 * * *$ & $0.130 * * *$ & $-0.540 * * *$ & $0.126 * * *$ & $-0.530 * * *$ \\
\hline & $(0.028)$ & $(0.119)$ & $(0.028)$ & $(0.119)$ & $(0.028)$ & $(0.119)$ & $(0.028)$ & $(0.119)$ & $(0.028)$ & $(0.119)$ \\
\hline \multirow[t]{2}{*}{ Agriculture \&Vet } & 0.020 & $-0.425 * *$ & 0.023 & $-0.450 * *$ & 0.018 & $-0.433 * *$ & 0.020 & $-0.453 * *$ & 0.021 & $-0.435^{* *}$ \\
\hline & $(0.044)$ & $(0.188)$ & $(0.044)$ & $(0.187)$ & $(0.044)$ & $(0.188)$ & $(0.045)$ & $(0.187)$ & $(0.044)$ & $(0.188)$ \\
\hline \multirow[t]{2}{*}{ Health } & $0.069^{* *}$ & -0.219 & $0.070 * *$ & $-0.234 *$ & $0.069 * *$ & $-0.230 *$ & $0.072 * *$ & $-0.233^{*}$ & $0.071 * *$ & $-0.234^{*}$ \\
\hline & $(0.030)$ & $(0.137)$ & $(0.030)$ & $(0.137)$ & $(0.029)$ & $(0.138)$ & $(0.030)$ & $(0.136)$ & $(0.030)$ & $(0.137)$ \\
\hline \multirow[t]{2}{*}{ Services } & 0.104 & $-0.608 *$ & 0.105 & $-0.629 *$ & 0.083 & $-0.634 *$ & 0.101 & $-0.620 *$ & 0.103 & $-0.659^{*}$ \\
\hline & $(0.107)$ & $(0.369)$ & $(0.107)$ & $(0.365)$ & $(0.107)$ & $(0.369)$ & $(0.108)$ & $(0.367)$ & $(0.107)$ & $(0.369)$ \\
\hline \multirow[t]{2}{*}{ Father w/ tertiary educ. } & & $-0.220 * * *$ & & $-0.213^{* * *}$ & & $-0.209 * * *$ & & $-0.214 * * *$ & & $-0.223 * * *$ \\
\hline & & $(0.079)$ & & $(0.079)$ & & $(0.079)$ & & $(0.079)$ & & $(0.079)$ \\
\hline \multirow[t]{2}{*}{ Constant } & $1.718 * * *$ & $3.066 * * *$ & $1.706^{* * *}$ & $2.847 * * *$ & $1.661 * * *$ & $3.047 * * *$ & $1.767 * * *$ & $2.824 * * *$ & $1.686^{* * *}$ & $3.162 * * *$ \\
\hline & $(0.116)$ & $(0.542)$ & $(0.119)$ & $(0.546)$ & $(0.115)$ & $(0.547)$ & $(0.123)$ & $(0.566)$ & $(0.120)$ & $(0.566)$ \\
\hline Observations & 2154 & & 2154 & & 2154 & & 2154 & & 2154 & \\
\hline Censored Obs. & 152 & & 152 & & 152 & & 152 & & 152 & \\
\hline Sigma & .389 & & .389 & & .387 & & .39 & & .388 & \\
\hline Rho & .887 & & .888 & & .885 & & .888 & & .883 & \\
\hline Lambda & .345 & & .346 & & .342 & & .346 & & .343 & \\
\hline
\end{tabular}


Table 3d. Heckman selection model, benchmark model.

\begin{tabular}{|c|c|c|c|c|c|c|c|c|c|c|}
\hline & \multicolumn{2}{|c|}{ Willingness to question ideas } & \multicolumn{2}{|c|}{ Ability to present } & \multicolumn{2}{|c|}{ Ability to write } & \multicolumn{2}{|c|}{ Foreign language } & \multicolumn{2}{|c|}{ Number of skills } \\
\hline & Ln(wage) & Selection & Ln(wage) & Selection & Ln(wage) & Selection & Ln(wage) & Selection & Ln(wage) & Selection \\
\hline \multirow[t]{2}{*}{ Own level of competence } & $0.019^{* * *}$ & 0.015 & $0.014 * *$ & 0.024 & 0.005 & 0.018 & $0.009^{*}$ & 0.005 & $0.009 * * *$ & -0.014 \\
\hline & $(0.007)$ & $(0.032)$ & $(0.006)$ & $(0.026)$ & $(0.007)$ & $(0.032)$ & $(0.005)$ & $(0.023)$ & $(0.002)$ & $(0.010)$ \\
\hline \multirow[t]{2}{*}{ Female } & $-0.092 * * *$ & 0.015 & $-0.093 * * *$ & 0.016 & $-0.094 * * *$ & 0.013 & $-0.093 * * *$ & 0.011 & $-0.102 * * *$ & 0.045 \\
\hline & $(0.019)$ & $(0.084)$ & $(0.019)$ & $(0.085)$ & $(0.019)$ & $(0.084)$ & $(0.019)$ & $(0.084)$ & $(0.019)$ & $(0.085)$ \\
\hline \multirow[t]{2}{*}{ Age } & -0.001 & $-0.032 * *$ & -0.001 & $-0.031 * *$ & -0.001 & $-0.031 * *$ & -0.001 & $-0.032 * *$ & $0.007 *$ & -0.025 \\
\hline & $(0.004)$ & $(0.016)$ & $(0.004)$ & $(0.016)$ & $(0.004)$ & $(0.016)$ & $(0.004)$ & $(0.016)$ & $(0.004)$ & $(0.016)$ \\
\hline \multirow[t]{2}{*}{ Long program } & $0.088 * * *$ & -0.131 & $0.084 * * *$ & -0.142 & $0.088 * * *$ & -0.136 & $0.085^{* * *}$ & -0.133 & $0.115 * * *$ & -0.160 \\
\hline & $(0.022)$ & $(0.097)$ & $(0.022)$ & $(0.097)$ & $(0.022)$ & $(0.097)$ & $(0.022)$ & $(0.097)$ & $(0.021)$ & $(0.103)$ \\
\hline \multirow[t]{2}{*}{ Grade in secondary edu. } & $0.056 * * *$ & $0.099 * *$ & $0.055 * * *$ & $0.096 * *$ & $0.056 * * *$ & $0.099 * *$ & $0.053 * * *$ & $0.098 * *$ & $0.054 * * *$ & 0.020 \\
\hline & $(0.010)$ & $(0.044)$ & $(0.010)$ & $(0.045)$ & $(0.010)$ & $(0.045)$ & $(0.010)$ & $(0.045)$ & $(0.010)$ & $(0.046)$ \\
\hline \multirow{2}{*}{ Experience } & $0.003 * * *$ & $-0.008 * * *$ & $0.003 * * *$ & $-0.008 * * *$ & $0.003 * * *$ & $-0.008 * * *$ & $0.003 * * *$ & $-0.008 * * *$ & $0.004 * * *$ & $-0.012 * * *$ \\
\hline & $(0.001)$ & $(0.003)$ & $(0.001)$ & $(0.003)$ & $(0.001)$ & $(0.003)$ & $(0.001)$ & $(0.003)$ & $(0.001)$ & $(0.003)$ \\
\hline \multicolumn{11}{|l|}{ Fields of study } \\
\hline \multirow[t]{2}{*}{ Education } & $0.129 * * *$ & 0.044 & $0.120 * * *$ & 0.024 & $0.128 * * *$ & 0.042 & $0.129 * * *$ & 0.040 & $0.171 * * *$ & -0.263 \\
\hline & $(0.032)$ & $(0.155)$ & $(0.032)$ & $(0.157)$ & $(0.032)$ & $(0.156)$ & $(0.032)$ & $(0.156)$ & $(0.032)$ & $(0.161)$ \\
\hline \multirow[t]{2}{*}{ Humanities } & -0.038 & -0.230 & -0.038 & -0.232 & -0.037 & -0.227 & -0.044 & -0.236 & 0.034 & -0.246 \\
\hline & $(0.037)$ & $(0.157)$ & $(0.037)$ & $(0.157)$ & $(0.037)$ & $(0.157)$ & $(0.037)$ & $(0.158)$ & $(0.037)$ & (0.164) \\
\hline \multirow[t]{2}{*}{ Science\&Maths } & 0.042 & 0.094 & 0.042 & 0.095 & $0.045^{*}$ & 0.102 & 0.040 & 0.093 & 0.029 & 0.035 \\
\hline & $(0.027)$ & $(0.142)$ & $(0.027)$ & $(0.142)$ & $(0.027)$ & $(0.142)$ & $(0.027)$ & $(0.142)$ & $(0.027)$ & $(0.143)$ \\
\hline \multirow[t]{2}{*}{ Engineering } & $0.126 * * *$ & $-0.539 * * *$ & $0.127 * * *$ & $-0.540 * * *$ & $0.129 * * *$ & $-0.539 * * *$ & $0.125 * * *$ & $-0.542 * * *$ & $0.203 * * *$ & $-0.573 * * *$ \\
\hline & $(0.028)$ & $(0.118)$ & $(0.028)$ & (0.119) & $(0.028)$ & $(0.118)$ & $(0.028)$ & (0.119) & $(0.028)$ & $(0.123)$ \\
\hline \multirow{2}{*}{ Agriculture\&Vet } & 0.017 & $-0.452 * *$ & 0.017 & $-0.456 * *$ & 0.020 & $-0.458 * *$ & 0.017 & $-0.459 * *$ & $0.089 * *$ & $-0.487 * *$ \\
\hline & $(0.044)$ & $(0.187)$ & $(0.044)$ & $(0.188)$ & $(0.045)$ & $(0.187)$ & $(0.044)$ & $(0.187)$ & $(0.044)$ & $(0.190)$ \\
\hline \multirow[t]{2}{*}{ Health } & $0.070 * *$ & $-0.232 *$ & $0.070 * *$ & $-0.239 *$ & $0.073 * *$ & $-0.232 *$ & $0.073 * *$ & $-0.235^{*}$ & $0.138 * * *$ & $-0.377 * * *$ \\
\hline & $(0.030)$ & $(0.136)$ & $(0.030)$ & $(0.137)$ & $(0.030)$ & $(0.136)$ & $(0.030)$ & $(0.136)$ & $(0.029)$ & $(0.138)$ \\
\hline \multirow[t]{2}{*}{ Services } & 0.093 & $-0.638 *$ & 0.104 & $-0.629 *$ & 0.102 & $-0.622 *$ & 0.091 & $-0.629 *$ & $0.283 * * *$ & $-0.620 *$ \\
\hline & $(0.107)$ & $(0.366)$ & $(0.107)$ & $(0.365)$ & $(0.108)$ & $(0.365)$ & $(0.108)$ & $(0.365)$ & $(0.107)$ & $(0.374)$ \\
\hline \multirow[t]{2}{*}{ Father w/ tertiary educ. } & & $-0.217 * * *$ & & $-0.218 * * *$ & & $-0.214 * * *$ & & $-0.209 * * *$ & & -0.045 \\
\hline & & $(0.079)$ & & $(0.079)$ & & $(0.079)$ & & $(0.080)$ & & $(0.081)$ \\
\hline \multirow[t]{2}{*}{ Constant } & $1.660 * * *$ & $2.823 * * *$ & $1.707 * * *$ & $2.777 * * *$ & $1.737 * * *$ & $2.769 * * *$ & $1.730 * * *$ & $2.876^{* * *}$ & $1.421 * * *$ & $3.338 * * *$ \\
\hline & $(0.120)$ & $(0.558)$ & $(0.115)$ & $(0.535)$ & $(0.119)$ & $(0.552)$ & $(0.115)$ & $(0.534)$ & $(0.116)$ & $(0.540)$ \\
\hline Observations & 2154 & & 2154 & & 2154 & & 2154 & & 2154 & \\
\hline Censored Obs. & 152 & & 152 & & 152 & & 152 & & 152 & \\
\hline Sigma & .389 & & .389 & & .39 & & .389 & & .386 & \\
\hline Rho & .891 & & .885 & & .889 & & .889 & & -.854 & \\
\hline Lambda & .347 & & .344 & & .347 & & .346 & & -.329 & \\
\hline
\end{tabular}


Table 4a. Heckman selection with required level of competences

\begin{tabular}{|c|c|c|c|c|c|c|c|c|c|c|}
\hline & \multicolumn{2}{|c|}{ Mastery of own field } & \multicolumn{2}{|c|}{ Knowledge other fields } & \multicolumn{2}{|c|}{ Analytical thinking } & \multicolumn{2}{|c|}{ Ability to learn } & \multicolumn{2}{|c|}{ Ability to negotiate } \\
\hline & Ln(wage) & Selection & Ln(wage) & Selection & Ln(wage) & Selection & Ln(wage) & Selection & Ln(wage) & Selection \\
\hline \multirow[t]{2}{*}{ Required level of competency } & $0.038 * * *$ & & $0.022 * * *$ & & $0.034 * * *$ & & $0.025 * * *$ & & $0.025 * * *$ & \\
\hline & $(0.006)$ & & $(0.006)$ & & $(0.006)$ & & $(0.006)$ & & $(0.005)$ & \\
\hline \multirow[t]{2}{*}{ Own level of competency } & -0.002 & -0.010 & -0.002 & $-0.057 *$ & $0.018 * *$ & -0.004 & -0.009 & -0.029 & -0.009 & -0.042 \\
\hline & $(0.008)$ & $(0.037)$ & $(0.008)$ & $(0.033)$ & $(0.008)$ & $(0.033)$ & $(0.009)$ & $(0.037)$ & $(0.007)$ & $(0.028)$ \\
\hline \multirow[t]{2}{*}{ Female } & $-0.095 * * *$ & 0.025 & $-0.098 * * *$ & -0.001 & $-0.085^{* * *}$ & 0.013 & $-0.096 * * *$ & 0.008 & $-0.097 * * *$ & 0.012 \\
\hline & $(0.018)$ & $(0.087)$ & $(0.019)$ & $(0.085)$ & $(0.019)$ & $(0.085)$ & $(0.019)$ & $(0.084)$ & $(0.019)$ & $(0.084)$ \\
\hline \multirow[t]{2}{*}{ Age } & 0.001 & $-0.035^{* *}$ & -0.001 & $-0.034 * *$ & 0.001 & $-0.037 * *$ & -0.000 & $-0.035^{* *}$ & -0.000 & $-0.033 * *$ \\
\hline & $(0.003)$ & $(0.016)$ & $(0.004)$ & $(0.016)$ & $(0.004)$ & $(0.016)$ & $(0.004)$ & $(0.016)$ & $(0.004)$ & $(0.016)$ \\
\hline \multirow[t]{2}{*}{ Long program } & $0.085^{* * *}$ & -0.134 & $0.091 * * *$ & -0.122 & $0.071 * * *$ & -0.110 & $0.085^{* * *}$ & -0.121 & $0.087 * * *$ & -0.146 \\
\hline & $(0.021)$ & (0.099) & $(0.021)$ & $(0.097)$ & $(0.021)$ & $(0.099)$ & $(0.021)$ & $(0.097)$ & $(0.021)$ & $(0.097)$ \\
\hline \multirow[t]{2}{*}{ Grade in secondary educ. } & $0.056 * * *$ & $0.091 * *$ & $0.057 * * *$ & $0.095^{* *}$ & $0.054 * * *$ & $0.102 * *$ & $0.057 * * *$ & $0.100 * *$ & $0.057 * * *$ & $0.094 * *$ \\
\hline & $(0.010)$ & $(0.046)$ & $(0.010)$ & $(0.045)$ & $(0.010)$ & $(0.045)$ & $(0.010)$ & $(0.045)$ & $(0.010)$ & $(0.044)$ \\
\hline \multirow[t]{2}{*}{ Experience } & $0.003 * * *$ & $-0.009 * * *$ & $0.003 * * *$ & $-0.009 * * *$ & $0.003 * * *$ & $-0.009 * * *$ & $0.003 * * *$ & $-0.008 * * *$ & $0.003 * * *$ & $-0.009 * * *$ \\
\hline & $(0.001)$ & $(0.003)$ & $(0.001)$ & $(0.003)$ & $(0.001)$ & $(0.003)$ & $(0.001)$ & $(0.003)$ & $(0.001)$ & $(0.003)$ \\
\hline \multirow[t]{2}{*}{ Education } & $0.129 * * *$ & -0.038 & $0.131 * * *$ & 0.012 & $0.136 * * *$ & 0.012 & $0.132 * * *$ & 0.018 & $0.136 * * *$ & 0.053 \\
\hline & $(0.031)$ & $(0.162)$ & $(0.032)$ & $(0.157)$ & $(0.032)$ & $(0.156)$ & $(0.032)$ & $(0.157)$ & $(0.032)$ & $(0.154)$ \\
\hline \multirow[t]{2}{*}{ Humanities } & -0.017 & -0.245 & -0.035 & -0.221 & -0.025 & -0.191 & -0.028 & -0.206 & -0.024 & -0.242 \\
\hline & $(0.036)$ & $(0.162)$ & $(0.037)$ & $(0.157)$ & $(0.036)$ & $(0.160)$ & $(0.037)$ & $(0.157)$ & $(0.037)$ & $(0.155)$ \\
\hline \multirow[t]{2}{*}{ Science\&Maths } & $0.048 *$ & 0.116 & 0.042 & 0.114 & 0.024 & 0.119 & 0.041 & 0.099 & $0.054 * *$ & 0.089 \\
\hline & $(0.026)$ & $(0.146)$ & $(0.027)$ & $(0.145)$ & $(0.027)$ & $(0.142)$ & $(0.027)$ & $(0.142)$ & $(0.027)$ & $(0.142)$ \\
\hline \multirow[t]{2}{*}{ Engineering } & $0.131 * * *$ & $-0.519 * * *$ & $0.124 * * *$ & $-0.515 * * *$ & $0.109 * * *$ & $-0.499 * * *$ & $0.129 * * *$ & $-0.526 * * *$ & $0.129 * * *$ & $-0.529 * * *$ \\
\hline & $(0.027)$ & $(0.122)$ & $(0.028)$ & $(0.119)$ & $(0.028)$ & $(0.120)$ & $(0.028)$ & $(0.119)$ & $(0.028)$ & $(0.119)$ \\
\hline \multirow[t]{2}{*}{ Agriculture \&Vet } & 0.025 & $-0.454 * *$ & 0.019 & $-0.434 * *$ & 0.013 & $-0.413 * *$ & 0.024 & $-0.448 * *$ & 0.023 & $-0.424 * *$ \\
\hline & $(0.044)$ & $(0.190)$ & $(0.044)$ & (0.189) & $(0.044)$ & $(0.188)$ & $(0.044)$ & $(0.188)$ & $(0.044)$ & $(0.188)$ \\
\hline \multirow[t]{2}{*}{ Health } & $0.054 *$ & $-0.263^{*}$ & $0.073 * *$ & $-0.252 *$ & $0.060 * *$ & $-0.232 *$ & $0.069 * *$ & $-0.232 *$ & $0.077 * * *$ & $-0.241 *$ \\
\hline & $(0.029)$ & $(0.141)$ & $(0.029)$ & $(0.137)$ & $(0.029)$ & $(0.138)$ & $(0.030)$ & $(0.137)$ & $(0.029)$ & $(0.136)$ \\
\hline \multirow[t]{2}{*}{ Services } & 0.113 & $-0.703 *$ & 0.112 & $-0.638 *$ & 0.091 & $-0.614 *$ & 0.101 & $-0.656^{*}$ & 0.090 & -0.576 \\
\hline & $(0.106)$ & $(0.372)$ & $(0.107)$ & $(0.367)$ & $(0.106)$ & $(0.365)$ & $(0.107)$ & $(0.365)$ & $(0.107)$ & $(0.367)$ \\
\hline \multirow[t]{2}{*}{ Father w/ tertiary educ. } & & $-0.217 * * *$ & & $-0.225 * * *$ & & $-0.207 * * *$ & & $-0.207 * * *$ & & $-0.207 * * *$ \\
\hline & & $(0.083)$ & & $(0.080)$ & & $(0.079)$ & & $(0.080)$ & & $(0.079)$ \\
\hline \multirow[t]{2}{*}{ Constant } & $1.542 * * *$ & $3.087 * * *$ & $1.670 * * *$ & $3.232 * * *$ & $1.476 * * *$ & $3.061 * * *$ & $1.657 * * *$ & $3.143 * * *$ & $1.666 * * *$ & $3.161 * * *$ \\
\hline & $(0.119)$ & $(0.557)$ & $(0.117)$ & $(0.551)$ & $(0.118)$ & $(0.552)$ & $(0.126)$ & $(0.583)$ & $(0.117)$ & $(0.539)$ \\
\hline Observations & 2154 & & 2154 & & 2154 & & 2154 & & 2154 & \\
\hline Censored Obs. & 152 & & 152 & & 152 & & 152 & & 152 & \\
\hline Sigma & .38 & & .386 & & .382 & & .388 & & .387 & \\
\hline Rho & .846 & & .877 & & .886 & & .887 & & .891 & \\
\hline Lambda & .322 & & .339 & & .339 & & .344 & & .345 & \\
\hline
\end{tabular}


Table 4b. Heckman selection with required level of competences

\begin{tabular}{|c|c|c|c|c|c|c|c|c|c|c|}
\hline & \multicolumn{2}{|c|}{ Ability to work under pressure } & \multicolumn{2}{|c|}{ Alertness to opportunities } & \multicolumn{2}{|c|}{ Ability to coordinate } & \multicolumn{2}{|c|}{ Ability to use time efficiently } & \multicolumn{2}{|c|}{ Ability to work with others } \\
\hline & Ln(wage) & Selection & Ln(wage) & Selection & Ln(wage) & Selection & Ln(wage) & Selection & Ln(wage) & Selection \\
\hline \multirow[t]{2}{*}{ Required level of competence } & 0.009 & & $0.013 * *$ & & $0.037 * * *$ & & $0.031 * * *$ & & $0.023 * * *$ & \\
\hline & $(0.006)$ & & $(0.005)$ & & $(0.006)$ & & $(0.006)$ & & $(0.006)$ & \\
\hline \multirow[t]{2}{*}{ Own level of competence } & -0.003 & $-0.062 *$ & -0.004 & $-0.107 * * *$ & -0.013 & -0.045 & $-0.023 * * *$ & -0.033 & 0.003 & $0.074 * *$ \\
\hline & $(0.008)$ & $(0.032)$ & $(0.007)$ & $(0.031)$ & $(0.008)$ & $(0.033)$ & $(0.008)$ & $(0.033)$ & $(0.008)$ & $(0.029)$ \\
\hline \multirow[t]{2}{*}{ Female } & $-0.095 * * *$ & 0.011 & $-0.098 * * *$ & 0.007 & $-0.101 * * *$ & 0.030 & $-0.097 * * *$ & 0.031 & $-0.102 * * *$ & -0.003 \\
\hline & $(0.019)$ & $(0.085)$ & $(0.019)$ & $(0.084)$ & $(0.019)$ & $(0.086)$ & $(0.019)$ & $(0.086)$ & $(0.019)$ & $(0.085)$ \\
\hline \multirow[t]{2}{*}{ Age } & -0.001 & $-0.034 * *$ & -0.000 & $-0.031 * *$ & 0.000 & $-0.037 * *$ & 0.000 & $-0.035 * *$ & -0.000 & $-0.032 * *$ \\
\hline & $(0.004)$ & $(0.016)$ & $(0.004)$ & $(0.016)$ & $(0.004)$ & $(0.016)$ & $(0.004)$ & $(0.016)$ & $(0.004)$ & $(0.016)$ \\
\hline \multirow[t]{2}{*}{ Long program } & $0.088 * * *$ & -0.123 & $0.085^{* * *}$ & -0.099 & $0.086 * * *$ & -0.124 & $0.085 * * *$ & -0.135 & $0.089 * * *$ & -0.121 \\
\hline & $(0.022)$ & $(0.098)$ & $(0.022)$ & $(0.097)$ & $(0.021)$ & $(0.099)$ & $(0.021)$ & $(0.098)$ & $(0.021)$ & $(0.098)$ \\
\hline \multirow[t]{2}{*}{ Grade secondary educ. } & $0.057 * * *$ & $0.097 * *$ & $0.058 * * *$ & $0.092 * *$ & $0.057 * * *$ & $0.095^{* *}$ & $0.058 * * *$ & $0.097 * *$ & $0.057 * * *$ & $0.099 * *$ \\
\hline & $(0.010)$ & $(0.045)$ & $(0.010)$ & $(0.044)$ & $(0.010)$ & $(0.045)$ & $(0.010)$ & $(0.045)$ & $(0.010)$ & $(0.045)$ \\
\hline \multirow[t]{2}{*}{ Experience } & $0.003 * * *$ & $-0.008 * * *$ & $0.003 * * *$ & $-0.008 * * *$ & $0.003 * * *$ & $-0.008 * * *$ & $0.003 * * *$ & $-0.009 * * *$ & $0.003 * * *$ & $-0.008 * * *$ \\
\hline & $(0.001)$ & $(0.003)$ & $(0.001)$ & $(0.003)$ & $(0.001)$ & $(0.003)$ & $(0.001)$ & $(0.003)$ & $(0.001)$ & $(0.003)$ \\
\hline \multirow[t]{2}{*}{ Education } & $0.132 * * *$ & 0.029 & $0.132 * * *$ & 0.085 & $0.130 * * *$ & -0.001 & $0.129 * * *$ & 0.014 & $0.122 * * *$ & 0.010 \\
\hline & $(0.032)$ & $(0.158)$ & $(0.032)$ & $(0.155)$ & $(0.032)$ & $(0.159)$ & $(0.032)$ & $(0.158)$ & $(0.032)$ & $(0.158)$ \\
\hline \multirow[t]{2}{*}{ Humanities } & -0.033 & -0.193 & -0.032 & -0.198 & -0.028 & -0.254 & -0.027 & -0.224 & -0.033 & -0.258 \\
\hline & $(0.037)$ & $(0.159)$ & $(0.037)$ & $(0.154)$ & $(0.036)$ & $(0.157)$ & $(0.037)$ & $(0.158)$ & $(0.037)$ & $(0.157)$ \\
\hline \multirow[t]{2}{*}{ Science\&Maths } & $0.045^{*}$ & 0.095 & $0.046^{*}$ & 0.106 & $0.049 *$ & 0.096 & $0.044 *$ & 0.110 & $0.045^{*}$ & 0.091 \\
\hline & $(0.027)$ & $(0.142)$ & $(0.027)$ & $(0.142)$ & $(0.027)$ & $(0.144)$ & $(0.027)$ & $(0.144)$ & $(0.027)$ & $(0.144)$ \\
\hline \multirow[t]{2}{*}{ Engineering } & $0.129 * * *$ & $-0.537 * * *$ & $0.126^{* * * *}$ & $-0.508 * * *$ & $0.122 * * *$ & $-0.533 * * *$ & $0.128 * * *$ & $-0.537 * * *$ & $0.125^{* * *}$ & $-0.555^{* * *}$ \\
\hline & $(0.028)$ & $(0.119)$ & $(0.028)$ & $(0.118)$ & $(0.028)$ & $(0.120)$ & $(0.028)$ & $(0.120)$ & $(0.028)$ & $(0.120)$ \\
\hline \multirow[t]{2}{*}{ Agriculture\&Vet } & 0.024 & $-0.443 * *$ & 0.020 & $-0.394 * *$ & 0.014 & $-0.475^{* *}$ & 0.021 & $-0.456^{* *}$ & 0.021 & $-0.491 * * *$ \\
\hline & $(0.044)$ & $(0.189)$ & $(0.044)$ & $(0.190)$ & $(0.044)$ & $(0.187)$ & $(0.044)$ & $(0.188)$ & $(0.044)$ & $(0.188)$ \\
\hline \multirow[t]{2}{*}{ Health } & $0.072 * *$ & $-0.240 *$ & $0.073 * *$ & -0.190 & $0.073 * *$ & $-0.257 *$ & $0.075 * *$ & $-0.245^{*}$ & $0.060 * *$ & $-0.276 * *$ \\
\hline & $(0.030)$ & $(0.139)$ & $(0.030)$ & $(0.135)$ & $(0.029)$ & $(0.140)$ & $(0.029)$ & $(0.138)$ & $(0.030)$ & $(0.138)$ \\
\hline \multirow[t]{2}{*}{ Services } & 0.105 & $-0.673^{*}$ & 0.097 & $-0.624 *$ & 0.101 & $-0.664 *$ & 0.113 & $-0.670^{*}$ & 0.110 & $-0.645^{*}$ \\
\hline & $(0.107)$ & $(0.368)$ & $(0.107)$ & $(0.363)$ & $(0.106)$ & $(0.371)$ & $(0.107)$ & $(0.366)$ & $(0.107)$ & $(0.367)$ \\
\hline \multirow{2}{*}{ Father w/ tertiary educ. } & & $-0.209 * * *$ & & $-0.224 * * *$ & & $-0.210 * * *$ & & $-0.212 * * *$ & & $-0.207 * *$ \\
\hline & & $(0.080)$ & & $(0.077)$ & & $(0.080)$ & & $(0.080)$ & & $(0.081)$ \\
\hline \multirow{2}{*}{ Constant } & $1.725 * * *$ & $3.270 * * *$ & $1.712 * * *$ & $3.344 * * *$ & $1.617 * * *$ & $3.301 * * *$ & $1.678 * * *$ & $3.173 * * *$ & $1.595 * * *$ & $2.488 * * *$ \\
\hline & $(0.121)$ & $(0.563)$ & $(0.118)$ & $(0.538)$ & $(0.119)$ & $(0.567)$ & $(0.124)$ & $(0.577)$ & $(0.122)$ & $(0.562)$ \\
\hline Observations & 2154 & & 2154 & & 2154 & & 2154 & & 2154 & \\
\hline Censored Obs. & 152 & & 152 & & 152 & & 152 & & 152 & \\
\hline Sigma & .388 & & .389 & & .383 & & .386 & & .386 & \\
\hline Rho & .878 & & .901 & & .872 & & .876 & & .878 & \\
\hline Lambda & .341 & & .351 & & .334 & & .338 & & .339 & \\
\hline
\end{tabular}


Table 4c. Heckman selection with required level of competences

\begin{tabular}{|c|c|c|c|c|c|c|c|c|c|c|}
\hline & \multicolumn{2}{|c|}{ Ability to mobilize others } & \multicolumn{2}{|c|}{ Ability to make meaning clear } & \multicolumn{2}{|c|}{ Ability to assert authority } & \multicolumn{2}{|c|}{ Ability to use computers } & \multicolumn{2}{|c|}{ Ability to come up with ideas } \\
\hline & Ln(wage) & Selection & Ln(wage) & Selection & Ln(wage) & Selection & Ln(wage) & Selection & Ln(wage) & Selection \\
\hline \multirow[t]{2}{*}{ Required level of competence } & $0.034 * * *$ & & $0.050 * * *$ & & $0.037 * * *$ & & 0.001 & & $0.034 * * *$ & \\
\hline & $(0.005)$ & & $(0.007)$ & & $(0.005)$ & & $(0.006)$ & & $(0.006)$ & \\
\hline \multirow[t]{2}{*}{ Own level of competence } & -0.012 & -0.032 & -0.010 & -0.019 & 0.005 & -0.004 & -0.001 & 0.007 & -0.005 & -0.031 \\
\hline & $(0.007)$ & $(0.029)$ & $(0.008)$ & $(0.037)$ & $(0.007)$ & $(0.029)$ & $(0.008)$ & $(0.033)$ & $(0.008)$ & $(0.035)$ \\
\hline \multirow[t]{2}{*}{ Female } & $-0.096 * * *$ & 0.007 & $-0.113 * * *$ & 0.042 & $-0.098 * * *$ & 0.023 & $-0.094 * * *$ & 0.013 & $-0.099 * * *$ & 0.013 \\
\hline & $(0.019)$ & $(0.085)$ & $(0.018)$ & $(0.087)$ & $(0.018)$ & $(0.085)$ & (0.019) & $(0.085)$ & $(0.019)$ & $(0.086)$ \\
\hline \multirow[t]{2}{*}{ Age } & -0.000 & $-0.035 * *$ & $0.008 * *$ & $-0.028 *$ & -0.000 & $-0.037 * *$ & -0.001 & $-0.031 * *$ & 0.001 & $-0.037 * *$ \\
\hline & $(0.004)$ & $(0.016)$ & $(0.003)$ & $(0.016)$ & $(0.003)$ & $(0.016)$ & $(0.004)$ & $(0.016)$ & $(0.004)$ & $(0.016)$ \\
\hline \multirow{2}{*}{ Long program } & $0.085 * * *$ & -0.127 & $0.103 * * *$ & -0.164 & $0.085 * * *$ & -0.121 & $0.089 * * *$ & -0.133 & $0.084 * * *$ & -0.136 \\
\hline & $(0.021)$ & $(0.098)$ & $(0.021)$ & $(0.104)$ & $(0.021)$ & $(0.099)$ & $(0.022)$ & $(0.097)$ & $(0.021)$ & $(0.099)$ \\
\hline \multirow[t]{2}{*}{ Grade secondary educ. } & $0.056 * * *$ & $0.096 * *$ & $0.057 * * *$ & 0.023 & $0.057 * * *$ & $0.089 * *$ & $0.057 * * *$ & $0.100 * *$ & $0.055 * * *$ & $0.093 * *$ \\
\hline & $(0.010)$ & $(0.045)$ & $(0.010)$ & $(0.046)$ & $(0.010)$ & $(0.045)$ & $(0.010)$ & $(0.045)$ & $(0.010)$ & $(0.045)$ \\
\hline \multirow[t]{2}{*}{ Experience } & $0.003 * * *$ & $-0.008 * * *$ & $0.004 * * *$ & $-0.012 * * *$ & $0.003 * * *$ & $-0.008 * * *$ & $0.003^{* * *}$ & $-0.008 * * *$ & $0.003 * * *$ & $-0.008 * * *$ \\
\hline & $(0.001)$ & $(0.003)$ & $(0.001)$ & $(0.003)$ & $(0.001)$ & $(0.003)$ & $(0.001)$ & $(0.003)$ & $(0.001)$ & $(0.003)$ \\
\hline \multirow[t]{2}{*}{ Education } & $0.122 * * *$ & 0.046 & $0.163 * * *$ & $-0.274^{*}$ & $0.106 * * *$ & 0.023 & $0.129 * * *$ & 0.047 & $0.121 * * *$ & 0.013 \\
\hline & $(0.032)$ & $(0.158)$ & $(0.031)$ & $(0.165)$ & $(0.031)$ & $(0.162)$ & $(0.032)$ & $(0.157)$ & $(0.032)$ & $(0.160)$ \\
\hline \multirow[t]{2}{*}{ Humanities } & -0.032 & -0.255 & 0.032 & -0.274 & -0.039 & $-0.290 *$ & -0.035 & -0.225 & -0.035 & -0.228 \\
\hline & $(0.036)$ & $(0.155)$ & $(0.036)$ & $(0.168)$ & $(0.036)$ & $(0.157)$ & $(0.037)$ & $(0.157)$ & $(0.036)$ & $(0.158)$ \\
\hline \multirow[t]{2}{*}{ Science\&Maths } & $0.052 *$ & 0.101 & 0.034 & 0.043 & $0.048^{*}$ & 0.111 & 0.044 & 0.099 & 0.036 & 0.132 \\
\hline & $(0.027)$ & $(0.142)$ & $(0.026)$ & $(0.146)$ & $(0.026)$ & $(0.143)$ & $(0.027)$ & $(0.142)$ & $(0.027)$ & $(0.145)$ \\
\hline \multirow[t]{2}{*}{ Engineering } & $0.122 * * *$ & $-0.530 * * *$ & $0.198 * * *$ & $-0.569 * * *$ & $0.115^{* * *}$ & $-0.513 * * *$ & $0.130 * * *$ & $-0.540 * * *$ & $0.116^{* * *}$ & $-0.525 * * *$ \\
\hline & $(0.028)$ & $(0.120)$ & $(0.027)$ & $(0.125)$ & $(0.027)$ & $(0.121)$ & $(0.028)$ & $(0.119)$ & $(0.028)$ & $(0.120)$ \\
\hline \multirow[t]{2}{*}{ Agriculture \&Vet } & 0.019 & $-0.436 * *$ & $0.090 * *$ & $-0.489 * *$ & 0.009 & $-0.425 * *$ & 0.020 & $-0.454 * *$ & 0.018 & $-0.431 * *$ \\
\hline & $(0.044)$ & $(0.187)$ & $(0.044)$ & $(0.192)$ & $(0.043)$ & $(0.188)$ & $(0.045)$ & $(0.187)$ & $(0.044)$ & $(0.189)$ \\
\hline \multirow[t]{2}{*}{ Health } & $0.064 * *$ & -0.219 & $0.122 * * *$ & $-0.372 * * *$ & $0.061 * *$ & -0.224 & $0.072 * *$ & $-0.233^{*}$ & $0.069 * *$ & $-0.233^{*}$ \\
\hline & $(0.029)$ & $(0.138)$ & $(0.029)$ & $(0.140)$ & $(0.029)$ & $(0.141)$ & $(0.030)$ & $(0.136)$ & $(0.029)$ & $(0.139)$ \\
\hline \multirow[t]{2}{*}{ Services } & 0.100 & $-0.603 *$ & $0.249 * *$ & $-0.651 *$ & 0.075 & $-0.655^{*}$ & 0.101 & $-0.620 *$ & 0.094 & $-0.694 *$ \\
\hline & $(0.106)$ & $(0.366)$ & $(0.106)$ & $(0.382)$ & $(0.105)$ & $(0.368)$ & $(0.108)$ & $(0.367)$ & $(0.106)$ & $(0.372)$ \\
\hline \multirow[t]{2}{*}{ Father w/ tertiary educ. } & & $-0.189 * *$ & & -0.064 & & $-0.190 * *$ & & $-0.214 * * *$ & & $-0.209 * * *$ \\
\hline & & $(0.080)$ & & $(0.083)$ & & $(0.081)$ & & $(0.079)$ & & $(0.080)$ \\
\hline \multirow[t]{2}{*}{ Constant } & $1.653 * * *$ & $3.137 * * *$ & $1.285 * * *$ & $3.324 * * *$ & $1.550 * * *$ & $3.072 * * *$ & $1.765^{* * *}$ & $2.826^{* * * *}$ & $1.587 * * *$ & $3.240 * * *$ \\
\hline & $(0.116)$ & $(0.550)$ & $(0.119)$ & $(0.553)$ & $(0.114)$ & $(0.556)$ & $(0.123)$ & $(0.566)$ & $(0.120)$ & $(0.570)$ \\
\hline Observations & 2154 & & 2154 & & 2154 & & 2154 & & 2154 & \\
\hline Censored Obs. & 152 & & 152 & & 152 & & 152 & & 152 & \\
\hline Sigma & .384 & & .378 & & .38 & & .39 & & .383 & \\
\hline Rho & .882 & & -.822 & & .869 & & .888 & & .868 & \\
\hline Lambda & .339 & & -.311 & & .33 & & .346 & & .333 & \\
\hline
\end{tabular}

$* \mathrm{p}<0.10, * * \mathrm{p}<0.05, * * * \mathrm{p}<0.01$ 
Table 4d. Heckman selection with required level of competences

\begin{tabular}{|c|c|c|c|c|c|c|c|c|c|c|}
\hline & \multicolumn{2}{|c|}{ Willingness to question ideas } & \multicolumn{2}{|c|}{ Ability to present } & \multicolumn{2}{|c|}{ Ability to write } & \multicolumn{2}{|c|}{ Foreign language } & \multicolumn{2}{|c|}{ Number of skills } \\
\hline & Ln(wage) & Selection & Ln(wage) & Selection & Ln(wage) & Selection & Ln(wage) & Selection & Ln(wage) & Selection \\
\hline \multirow{2}{*}{ Required level of competence } & $0.031 * * *$ & & $0.029 * * *$ & & $0.037 * * *$ & & $0.016^{* * *}$ & & $0.012 * * *$ & \\
\hline & $(0.006)$ & & $(0.005)$ & & $(0.006)$ & & $(0.004)$ & & $(0.002)$ & \\
\hline \multirow[t]{2}{*}{ Own level of competence } & 0.003 & 0.017 & -0.005 & 0.024 & $-0.020 * *$ & 0.020 & 0.000 & 0.004 & -0.000 & -0.006 \\
\hline & $(0.008)$ & $(0.032)$ & $(0.006)$ & $(0.026)$ & $(0.008)$ & $(0.032)$ & $(0.005)$ & $(0.023)$ & $(0.002)$ & $(0.010)$ \\
\hline \multirow[t]{2}{*}{ Female } & $-0.097 * * *$ & 0.006 & $-0.096 * * *$ & 0.021 & $-0.099 * * *$ & 0.005 & $-0.093 * * *$ & 0.009 & $-0.101 * * *$ & 0.012 \\
\hline & $(0.019)$ & $(0.086)$ & $(0.019)$ & $(0.085)$ & $(0.019)$ & $(0.086)$ & $(0.019)$ & $(0.085)$ & $(0.019)$ & $(0.086)$ \\
\hline \multirow[t]{2}{*}{ Age } & 0.000 & $-0.033 * *$ & -0.000 & $-0.032 * *$ & 0.000 & $-0.033^{* *}$ & -0.000 & $-0.033 * *$ & 0.001 & $-0.036 * *$ \\
\hline & $(0.004)$ & $(0.016)$ & $(0.004)$ & $(0.016)$ & $(0.003)$ & $(0.016)$ & $(0.004)$ & $(0.016)$ & $(0.003)$ & $(0.016)$ \\
\hline \multirow[t]{2}{*}{ Long program } & $0.083 * * *$ & -0.138 & $0.075 * * *$ & -0.142 & $0.079 * * *$ & -0.136 & $0.081 * * *$ & -0.121 & $0.079 * * *$ & -0.125 \\
\hline & $(0.021)$ & $(0.098)$ & $(0.021)$ & $(0.098)$ & $(0.021)$ & $(0.099)$ & $(0.022)$ & $(0.098)$ & $(0.021)$ & $(0.099)$ \\
\hline \multirow[t]{2}{*}{ Grade secondary educ. } & $0.056^{* * *}$ & $0.092 * *$ & $0.055 * * *$ & $0.094 * *$ & $0.058 * * *$ & $0.095 * *$ & $0.053 * * *$ & $0.095 * *$ & $0.056 * * *$ & $0.089 * *$ \\
\hline & $(0.010)$ & $(0.045)$ & $(0.010)$ & $(0.045)$ & $(0.010)$ & $(0.046)$ & $(0.010)$ & $(0.045)$ & $(0.010)$ & $(0.045)$ \\
\hline \multirow[t]{2}{*}{ Experience } & $0.003 * * *$ & $-0.008 * * *$ & $0.003 * * *$ & $-0.008 * * *$ & $0.003 * * *$ & $-0.009 * * *$ & $0.003 * * *$ & $-0.008 * * *$ & $0.003 * * *$ & $-0.009 * * *$ \\
\hline & $(0.001)$ & $(0.003)$ & $(0.001)$ & $(0.003)$ & $(0.001)$ & $(0.003)$ & $(0.001)$ & $(0.003)$ & $(0.001)$ & $(0.003)$ \\
\hline \multirow[t]{2}{*}{ Education } & $0.127 * * *$ & 0.033 & $0.108 * * *$ & 0.002 & $0.131 * * *$ & -0.001 & $0.129 * * *$ & 0.029 & $0.129 * * *$ & 0.012 \\
\hline & $(0.032)$ & $(0.159)$ & $(0.032)$ & $(0.161)$ & $(0.032)$ & $(0.162)$ & $(0.032)$ & $(0.157)$ & $(0.031)$ & $(0.161)$ \\
\hline \multirow[t]{2}{*}{ Humanities } & -0.030 & -0.231 & -0.031 & -0.247 & -0.016 & -0.245 & -0.046 & -0.238 & -0.024 & -0.243 \\
\hline & $(0.036)$ & $(0.159)$ & $(0.036)$ & $(0.158)$ & $(0.036)$ & $(0.159)$ & $(0.037)$ & $(0.157)$ & $(0.036)$ & $(0.158)$ \\
\hline \multirow[t]{2}{*}{ Science\&Maths } & 0.041 & 0.123 & 0.038 & 0.129 & 0.039 & 0.119 & 0.033 & 0.117 & $0.045^{*}$ & 0.113 \\
\hline & $(0.027)$ & $(0.144)$ & $(0.027)$ & $(0.145)$ & $(0.027)$ & $(0.146)$ & $(0.027)$ & $(0.143)$ & $(0.026)$ & $(0.145)$ \\
\hline \multirow[t]{2}{*}{ Engineering } & $0.115^{* * *}$ & $-0.542 * * *$ & $0.119 * * *$ & $-0.531 * * *$ & $0.123 * * *$ & $-0.531 * * *$ & $0.116^{* * *}$ & $-0.531 * * *$ & $0.112 * * *$ & $-0.514 * * *$ \\
\hline & $(0.028)$ & $(0.120)$ & $(0.028)$ & $(0.120)$ & $(0.027)$ & $(0.120)$ & $(0.028)$ & $(0.119)$ & $(0.027)$ & $(0.121)$ \\
\hline \multirow[t]{2}{*}{ Agriculture \&Vet } & 0.012 & $-0.448 * *$ & 0.015 & $-0.459 * *$ & 0.012 & $-0.494 * * *$ & 0.022 & $-0.456 * *$ & 0.022 & $-0.424 * *$ \\
\hline & $(0.044)$ & $(0.187)$ & $(0.044)$ & $(0.188)$ & $(0.044)$ & $(0.187)$ & $(0.044)$ & $(0.187)$ & $(0.044)$ & $(0.190)$ \\
\hline \multirow[t]{2}{*}{ Health } & $0.064 * *$ & $-0.233^{*}$ & $0.068 * *$ & -0.227 & $0.080 * * *$ & $-0.236^{*}$ & $0.069 * *$ & -0.212 & $0.073 * *$ & -0.230 \\
\hline & $(0.029)$ & $(0.138)$ & $(0.029)$ & $(0.139)$ & $(0.029)$ & $(0.140)$ & $(0.030)$ & $(0.137)$ & $(0.029)$ & $(0.142)$ \\
\hline \multirow[t]{2}{*}{ Services } & 0.095 & $-0.660 *$ & 0.119 & $-0.643^{*}$ & 0.124 & $-0.681^{*}$ & 0.096 & $-0.614^{*}$ & 0.127 & $-0.685^{*}$ \\
\hline & $(0.106)$ & $(0.367)$ & $(0.106)$ & $(0.366)$ & $(0.106)$ & $(0.372)$ & $(0.107)$ & $(0.367)$ & $(0.106)$ & $(0.372)$ \\
\hline \multirow[t]{2}{*}{ Father w/ tertiary educ. } & & $-0.216^{* * *}$ & & $-0.208 * *$ & & $-0.191 * *$ & & $-0.205^{* *}$ & & $-0.202 * *$ \\
\hline & & $(0.080)$ & & $(0.081)$ & & $(0.083)$ & & $(0.081)$ & & $(0.081)$ \\
\hline \multirow[t]{2}{*}{ Constant } & $1.584 * * *$ & $2.870 * * *$ & $1.652 * * *$ & $2.800 * * *$ & $1.647 * * *$ & $2.840 * * *$ & $1.715^{* * *}$ & $2.903 * * *$ & $1.588 * * *$ & $3.149 * * *$ \\
\hline & $(0.119)$ & $(0.568)$ & $(0.114)$ & $(0.544)$ & $(0.118)$ & $(0.563)$ & $(0.114)$ & $(0.535)$ & $(0.115)$ & $(0.550)$ \\
\hline Observations & 2154 & & 2154 & & 2154 & & 2154 & & 2154 & \\
\hline Censored Obs. & 152 & & 152 & & 152 & & 152 & & 152 & \\
\hline Sigma & .384 & & .383 & & .382 & & .388 & & .381 & \\
\hline Rho & .874 & & .868 & & .859 & & .886 & & .859 & \\
\hline Lambda & .336 & & .333 & & .328 & & .343 & & .327 & \\
\hline
\end{tabular}

$* \mathrm{p}<0.10, * * \mathrm{p}<0.05, * * * \mathrm{p}<0.01$ 
Table 5a. Heckman selection with job characteristics

\begin{tabular}{|c|c|c|c|c|c|c|c|c|c|c|}
\hline & \multicolumn{2}{|c|}{ Mastery of own field } & \multicolumn{2}{|c|}{ Knowledge other fields } & \multicolumn{2}{|c|}{ Analytical thinking } & \multicolumn{2}{|c|}{ Ability to learn } & \multicolumn{2}{|c|}{ Ability to negotiate } \\
\hline & Ln(wage) & Selection & Ln(wage) & Selection & Ln(wage) & Selection & Ln(wage) & Selection & Ln(wage) & Selection \\
\hline \multirow[t]{2}{*}{ Required level of competence } & 0.036 *** & & $0.021 * * *$ & & $0.031 * * *$ & & $0.026 * * *$ & & $0.031 * * *$ & \\
\hline & $(0.006)$ & & $(0.006)$ & & $(0.006)$ & & $(0.006)$ & & $(0.005)$ & \\
\hline \multirow{2}{*}{ Own level of competence } & -0.006 & -0.020 & -0.004 & $-0.064 *$ & 0.012 & -0.002 & -0.010 & -0.027 & -0.007 & -0.051 \\
\hline & $(0.008)$ & $(0.042)$ & $(0.007)$ & $(0.035)$ & $(0.008)$ & $(0.035)$ & $(0.009)$ & $(0.042)$ & $(0.006)$ & $(0.031)$ \\
\hline \multirow[t]{2}{*}{ Grade secondary educ. } & $0.029 * * *$ & 0.046 & $0.030 * * *$ & 0.054 & $0.028 * * *$ & 0.060 & $0.030 * * *$ & 0.061 & $0.030 * * *$ & 0.048 \\
\hline & $(0.009)$ & $(0.052)$ & $(0.009)$ & $(0.050)$ & $(0.009)$ & $(0.050)$ & $(0.009)$ & $(0.050)$ & $(0.009)$ & $(0.050)$ \\
\hline \multirow[t]{2}{*}{ Education } & $0.088 * * *$ & $-0.300^{*}$ & $0.083 * * *$ & -0.230 & $0.088 * * *$ & -0.241 & $0.085 * * *$ & -0.238 & $0.084 * * *$ & -0.222 \\
\hline & $(0.031)$ & $(0.179)$ & $(0.031)$ & $(0.183)$ & $(0.031)$ & $(0.181)$ & $(0.031)$ & $(0.182)$ & $(0.031)$ & $(0.182)$ \\
\hline \multirow[t]{2}{*}{ Humanities } & -0.029 & $-0.406 * *$ & -0.051 & $-0.375^{* *}$ & -0.041 & $-0.375 * *$ & -0.045 & $-0.374 * *$ & -0.042 & $-0.394 * *$ \\
\hline & $(0.035)$ & $(0.175)$ & $(0.035)$ & $(0.172)$ & $(0.034)$ & $(0.172)$ & $(0.035)$ & $(0.171)$ & $(0.034)$ & $(0.172)$ \\
\hline \multirow[t]{2}{*}{ Science\&Maths } & $0.063 * * *$ & 0.113 & $0.057 * *$ & 0.134 & $0.043^{*}$ & 0.130 & $0.058 * *$ & 0.123 & $0.073 * * *$ & 0.111 \\
\hline & $(0.024)$ & $(0.158)$ & $(0.025)$ & $(0.157)$ & $(0.025)$ & $(0.157)$ & $(0.025)$ & $(0.155)$ & $(0.025)$ & $(0.157)$ \\
\hline \multirow[t]{2}{*}{ Engineering } & $0.173 * * *$ & $-0.513 * * *$ & $0.162 * * *$ & $-0.499 * * *$ & $0.149 * * *$ & $-0.495 * * *$ & $0.167 * * *$ & $-0.503 * * *$ & $0.169 * * *$ & $-0.506 * * *$ \\
\hline & $(0.027)$ & $(0.130)$ & $(0.027)$ & $(0.129)$ & $(0.027)$ & $(0.130)$ & $(0.027)$ & $(0.128)$ & $(0.026)$ & $(0.129)$ \\
\hline \multirow[t]{2}{*}{ Agriculture\&Vet } & 0.041 & $-0.481 * *$ & 0.030 & $-0.479 * *$ & 0.023 & $-0.481 * *$ & 0.033 & $-0.487 * *$ & 0.029 & $-0.479 * *$ \\
\hline & $(0.042)$ & $(0.202)$ & $(0.042)$ & $(0.201)$ & $(0.042)$ & $(0.200)$ & $(0.042)$ & $(0.200)$ & $(0.042)$ & $(0.200)$ \\
\hline \multirow[t]{2}{*}{ Health } & 0.035 & $-0.440 * * *$ & 0.041 & $-0.391 * *$ & 0.031 & $-0.390 * *$ & 0.039 & $-0.389 * *$ & 0.039 & $-0.407 * * *$ \\
\hline & $(0.029)$ & $(0.155)$ & $(0.029)$ & $(0.160)$ & $(0.029)$ & $(0.159)$ & $(0.029)$ & $(0.158)$ & $(0.029)$ & $(0.157)$ \\
\hline \multirow[t]{2}{*}{ Services } & 0.044 & $-0.880 * *$ & 0.030 & $-0.854 * *$ & 0.014 & $-0.852 * *$ & 0.018 & $-0.874 * *$ & -0.001 & $-0.847 * *$ \\
\hline & $(0.101)$ & $(0.392)$ & $(0.102)$ & $(0.389)$ & $(0.101)$ & $(0.389)$ & $(0.102)$ & $(0.386)$ & $(0.101)$ & $(0.390)$ \\
\hline \multirow[t]{2}{*}{ Public sector } & $0.210 * * *$ & & $0.215^{* * *}$ & & $0.212 * * *$ & & $0.216 * * *$ & & $0.236 * * *$ & \\
\hline & $(0.019)$ & & $(0.019)$ & & $(0.019)$ & & $(0.019)$ & & $(0.019)$ & \\
\hline \multirow[t]{2}{*}{ Permanent contract } & $0.160 * * *$ & & $0.161 * * *$ & & $0.161 * * *$ & & $0.162 * * *$ & & $0.154 * * *$ & \\
\hline & $(0.017)$ & & $(0.017)$ & & $(0.017)$ & & $(0.017)$ & & $(0.017)$ & \\
\hline \multirow{2}{*}{ Medium firm } & $0.100 * * *$ & & $0.096 * * *$ & & $0.095 * * *$ & & $0.097 * * *$ & & $0.096 * * *$ & \\
\hline & $(0.023)$ & & $(0.023)$ & & $(0.022)$ & & $(0.023)$ & & $(0.022)$ & \\
\hline \multirow[t]{2}{*}{ Large firm } & $0.139 * * *$ & & $0.135 * * *$ & & $0.128 * * *$ & & $0.132 * * *$ & & $0.135^{* * *}$ & \\
\hline & $(0.019)$ & & $(0.019)$ & & $(0.019)$ & & $(0.019)$ & & $(0.019)$ & \\
\hline Father w/ tertiary educ. & & $\begin{array}{c}-0.232 * * \\
(0.093)\end{array}$ & & $\begin{array}{c}-0.232 * * * \\
(0.090)\end{array}$ & & $\begin{array}{c}-0.227 * * \\
(0.090)\end{array}$ & & $\begin{array}{c}-0.231 * * \\
(0.090)\end{array}$ & & $\begin{array}{c}-0.229 * * \\
(0.090)\end{array}$ \\
\hline \multirow[t]{2}{*}{ Constant } & $1.516 * * *$ & $3.532 * * *$ & $1.652 * * *$ & $3.662 * * *$ & $1.497 * * *$ & $3.375 * * *$ & $1.631 * * *$ & $3.519 * * *$ & $1.596 * * *$ & $3.640 * * *$ \\
\hline & $(0.113)$ & $(0.585)$ & $(0.111)$ & $(0.568)$ & $(0.111)$ & $(0.567)$ & $(0.119)$ & $(0.605)$ & $(0.109)$ & $(0.557)$ \\
\hline Observations & 1997 & & 1997 & & 1997 & & 1997 & & 1997 & \\
\hline Censored Obs. & 152 & & 152 & & 152 & & 152 & & 152 & \\
\hline Sigma & .332 & & .342 & & .34 & & .343 & & .339 & \\
\hline Rho & .486 & & .652 & & .662 & & .669 & & .643 & \\
\hline Lambda & .161 & & .223 & & .225 & & .23 & & .218 & \\
\hline
\end{tabular}


Table 5b. Heckman selection with job characteristics

\begin{tabular}{|c|c|c|c|c|c|c|c|c|c|c|}
\hline & \multicolumn{2}{|c|}{ Ability to work under pressure } & \multicolumn{2}{|c|}{ Alertness to opportunities } & \multicolumn{2}{|c|}{ Ability to coordinate } & \multicolumn{2}{|c|}{ Ability to use time efficiently } & \multicolumn{2}{|c|}{ Ability to work with others } \\
\hline & Selection & Ln(wage) & Selection & Ln(wage) & Selection & & Selection & Ln(wage) & Selection & Ln(wage) \\
\hline \multirow[t]{2}{*}{ Required level of competence } & $0.013 * *$ & & $0.021 * * *$ & & $0.037 * * *$ & & $0.033 * * *$ & & $0.020 * * *$ & \\
\hline & $(0.006)$ & & $(0.005)$ & & $(0.006)$ & & $(0.006)$ & & $(0.006)$ & \\
\hline \multirow[t]{2}{*}{ Own level of competence } & 0.000 & $-0.077 * *$ & -0.006 & $-0.110 * * *$ & -0.009 & -0.059 & $-0.022 * * *$ & -0.043 & 0.004 & $0.087 * *$ \\
\hline & $(0.008)$ & $(0.036)$ & $(0.007)$ & $(0.035)$ & $(0.008)$ & $(0.036)$ & $(0.007)$ & $(0.036)$ & $(0.008)$ & $(0.034)$ \\
\hline \multirow[t]{2}{*}{ Grade secondary educ. } & $0.030 * * *$ & 0.058 & $0.031 * * *$ & 0.050 & $0.029 * * *$ & 0.060 & $0.031 * * *$ & 0.063 & $0.030 * * *$ & 0.054 \\
\hline & $(0.009)$ & $(0.050)$ & $(0.009)$ & $(0.050)$ & $(0.009)$ & $(0.050)$ & $(0.009)$ & $(0.049)$ & $(0.009)$ & $(0.051)$ \\
\hline \multirow[t]{2}{*}{ Education } & $0.085^{* * *}$ & -0.228 & $0.086^{* * *}$ & -0.200 & $0.079 * *$ & -0.226 & $0.078 * *$ & -0.213 & $0.075 * *$ & -0.285 \\
\hline & $(0.031)$ & $(0.185)$ & $(0.031)$ & $(0.187)$ & $(0.031)$ & $(0.178)$ & $(0.031)$ & $(0.179)$ & $(0.031)$ & $(0.186)$ \\
\hline \multirow[t]{2}{*}{ Humanities } & -0.052 & $-0.355^{* *}$ & -0.045 & $-0.371 * *$ & -0.051 & $-0.388 * *$ & -0.048 & $-0.373 * *$ & -0.050 & $-0.404 * *$ \\
\hline & $(0.035)$ & $(0.173)$ & $(0.035)$ & $(0.173)$ & $(0.034)$ & $(0.170)$ & $(0.035)$ & $(0.168)$ & $(0.035)$ & $(0.174)$ \\
\hline \multirow[t]{2}{*}{ Science\&Maths } & $0.062 * *$ & 0.118 & $0.062 * *$ & 0.127 & $0.065 * * *$ & 0.104 & $0.060 * *$ & 0.129 & $0.061 * *$ & 0.096 \\
\hline & $(0.025)$ & $(0.156)$ & $(0.025)$ & $(0.158)$ & $(0.025)$ & $(0.155)$ & $(0.025)$ & $(0.155)$ & $(0.025)$ & $(0.158)$ \\
\hline \multirow[t]{2}{*}{ Engineering } & $0.165 * * *$ & $-0.506 * * *$ & $0.166^{* * *}$ & $-0.491 * * *$ & $0.159 * * *$ & $-0.508 * * *$ & $0.163 * * *$ & $-0.509 * * *$ & $0.166^{* * *}$ & $-0.528 * * *$ \\
\hline & $(0.027)$ & $(0.128)$ & $(0.027)$ & $(0.129)$ & $(0.026)$ & $(0.128)$ & $(0.026)$ & $(0.127)$ & $(0.027)$ & $(0.129)$ \\
\hline \multirow[t]{2}{*}{ Agriculture \&Vet } & 0.035 & $-0.488 * *$ & 0.032 & $-0.457 * *$ & 0.024 & $-0.502 * *$ & 0.027 & $-0.484 * *$ & 0.033 & $-0.511 * *$ \\
\hline & $(0.042)$ & $(0.201)$ & $(0.042)$ & $(0.202)$ & $(0.042)$ & $(0.199)$ & $(0.042)$ & $(0.199)$ & $(0.042)$ & $(0.200)$ \\
\hline \multirow[t]{2}{*}{ Health } & 0.037 & $-0.389 * *$ & 0.041 & $-0.371 * *$ & 0.039 & $-0.406 * * *$ & 0.041 & $-0.364 * *$ & 0.032 & $-0.446 * * *$ \\
\hline & $(0.029)$ & $(0.158)$ & $(0.029)$ & $(0.161)$ & $(0.029)$ & $(0.156)$ & $(0.029)$ & $(0.158)$ & $(0.030)$ & $(0.159)$ \\
\hline \multirow[t]{2}{*}{ Services } & 0.024 & $-0.900 * *$ & 0.017 & $-0.876 * *$ & 0.014 & $-0.847 * *$ & 0.026 & $-0.859 * *$ & 0.037 & $-0.909 * *$ \\
\hline & $(0.102)$ & $(0.388)$ & $(0.102)$ & $(0.389)$ & $(0.101)$ & $(0.390)$ & $(0.102)$ & $(0.383)$ & $(0.102)$ & $(0.387)$ \\
\hline \multirow[t]{2}{*}{ Public sector } & $0.222 * * *$ & & $0.225^{* * *}$ & & $0.216^{* * *}$ & & $0.217 * * *$ & & $0.220 * * *$ & \\
\hline & $(0.019)$ & & $(0.020)$ & & $(0.019)$ & & $(0.019)$ & & $(0.019)$ & \\
\hline \multirow[t]{2}{*}{ Permanent contract } & $0.159 * * *$ & & $0.158 * * *$ & & $0.156^{* * *}$ & & $0.158 * * *$ & & $0.157 * * *$ & \\
\hline & $(0.017)$ & & $(0.017)$ & & $(0.017)$ & & $(0.017)$ & & $(0.017)$ & \\
\hline \multirow[t]{2}{*}{ Medium firm } & $0.094 * * *$ & & $0.097 * * *$ & & $0.098 * * *$ & & $0.095 * * *$ & & $0.091 * * *$ & \\
\hline & $(0.023)$ & & $(0.023)$ & & $(0.022)$ & & $(0.023)$ & & $(0.023)$ & \\
\hline \multirow[t]{2}{*}{ Large firm } & $0.131 * * *$ & & $0.137 * * *$ & & $0.139 * * *$ & & $0.133 * * *$ & & $0.128 * * *$ & \\
\hline & $(0.019)$ & & $(0.019)$ & & $(0.019)$ & & $(0.019)$ & & $(0.019)$ & \\
\hline \multirow[t]{2}{*}{ Father w/ tertiary educ. } & & $-0.224 * *$ & & $-0.237 * * *$ & & $-0.218 * *$ & & $-0.226 * *$ & & $-0.234 * *$ \\
\hline & & $(0.090)$ & & $(0.090)$ & & $(0.090)$ & & $(0.089)$ & & $(0.092)$ \\
\hline \multirow[t]{2}{*}{ Constant } & $1.660 * * *$ & $3.752 * * *$ & $1.644 * * *$ & $3.853 * * *$ & $1.572 * * *$ & $3.709 * * *$ & $1.641 * * *$ & $3.588 * * *$ & $1.583 * * *$ & $2.892 * * *$ \\
\hline & $(0.114)$ & $(0.584)$ & $(0.112)$ & $(0.572)$ & $(0.112)$ & $(0.574)$ & $(0.117)$ & $(0.581)$ & $(0.113)$ & $(0.570)$ \\
\hline Observations & 1997 & & 1997 & & 1997 & & 1997 & & 1997 & \\
\hline Censored Obs. & 152 & & 152 & & 152 & & 152 & & 152 & \\
\hline Sigma & .344 & & .341 & & .341 & & .345 & & .34 & \\
\hline Rho & .657 & & .645 & & .671 & & .712 & & .603 & \\
\hline Lambda & .226 & & .22 & & .229 & & .246 & & .205 & \\
\hline
\end{tabular}

Other controls included: female, age, long program, experience. $* \mathrm{p}<0.10, * * \mathrm{p}<0.05, * * * \mathrm{p}<0.01$ 
Table 5c. Heckman selection with job characteristics

\begin{tabular}{|c|c|c|c|c|c|c|c|c|c|c|}
\hline & \multicolumn{2}{|c|}{ Ability to mobilize others } & \multicolumn{2}{|c|}{ Ability to make meaning clear } & \multirow{2}{*}{\multicolumn{2}{|c|}{$\begin{array}{l}\text { Ability to assert authority } \\
\text { Ln(wage) }\end{array}$}} & \multicolumn{2}{|c|}{ Ability to use computers } & \multicolumn{2}{|c|}{ Ability to come up with ideas } \\
\hline & Ln(wage) & Selection & Ln(wage) & Selection & & & Ln(wage) & Selection & Ln(wage) & Selection \\
\hline \multirow[t]{2}{*}{ Required level of competence } & $0.032 * * *$ & & $0.042 * * *$ & & $0.032 * * *$ & & 0.004 & & $0.033 * * *$ & \\
\hline & $(0.005)$ & & $(0.006)$ & & $(0.005)$ & & $(0.006)$ & & $(0.006)$ & \\
\hline \multirow[t]{2}{*}{ Own level of competence } & -0.011 & -0.048 & -0.013 & -0.001 & 0.003 & -0.025 & -0.004 & 0.031 & -0.002 & -0.057 \\
\hline & $(0.007)$ & $(0.032)$ & $(0.008)$ & $(0.037)$ & $(0.006)$ & $(0.032)$ & $(0.008)$ & $(0.035)$ & $(0.008)$ & $(0.039)$ \\
\hline \multirow[t]{2}{*}{ Grade secondary educ. } & $0.030 * * *$ & 0.064 & $0.031 * * *$ & 0.059 & $0.031 * * *$ & 0.056 & $0.030 * * *$ & 0.059 & $0.028 * * *$ & 0.054 \\
\hline & $(0.009)$ & $(0.050)$ & $(0.009)$ & $(0.050)$ & $(0.009)$ & $(0.050)$ & $(0.009)$ & $(0.050)$ & $(0.009)$ & $(0.051)$ \\
\hline \multirow[t]{2}{*}{ Education } & $0.074 * *$ & -0.200 & $0.076 * *$ & -0.229 & $0.062 * *$ & -0.223 & $0.083 * * *$ & -0.209 & $0.077 * *$ & -0.242 \\
\hline & $(0.031)$ & $(0.183)$ & $(0.031)$ & $(0.183)$ & $(0.031)$ & $(0.185)$ & $(0.031)$ & $(0.184)$ & $(0.031)$ & $(0.183)$ \\
\hline \multirow[t]{2}{*}{ Humanities } & -0.054 & $-0.380 * *$ & -0.055 & $-0.388 * *$ & $-0.058^{*}$ & $-0.394 * *$ & -0.052 & $-0.386 * *$ & -0.051 & $-0.388 * *$ \\
\hline & $(0.034)$ & $(0.169)$ & $(0.034)$ & $(0.171)$ & $(0.034)$ & $(0.171)$ & $(0.035)$ & $(0.171)$ & $(0.034)$ & $(0.173)$ \\
\hline \multirow[t]{2}{*}{ Science\&Maths } & $0.068 * * *$ & 0.125 & $0.062 * *$ & 0.128 & $0.063^{* *}$ & 0.124 & $0.060 * *$ & 0.109 & $0.051 * *$ & 0.134 \\
\hline & $(0.025)$ & $(0.155)$ & $(0.025)$ & $(0.156)$ & $(0.025)$ & $(0.156)$ & $(0.025)$ & $(0.156)$ & $(0.025)$ & $(0.158)$ \\
\hline \multirow[t]{2}{*}{ Engineering } & $0.158 * * *$ & $-0.498 * * *$ & $0.158 * * *$ & $-0.506 * * *$ & $0.154 * * *$ & $-0.498 * * *$ & $0.167 * * *$ & $-0.522 * * *$ & $0.154 * * *$ & $-0.501 * * *$ \\
\hline & $(0.026)$ & $(0.128)$ & $(0.027)$ & $(0.128)$ & $(0.027)$ & $(0.129)$ & $(0.027)$ & $(0.128)$ & $(0.027)$ & $(0.129)$ \\
\hline \multirow[t]{2}{*}{ Agriculture \&Vet } & 0.029 & $-0.467 * *$ & 0.030 & $-0.488 * *$ & 0.019 & $-0.476^{* *}$ & 0.031 & $-0.489 * *$ & 0.031 & $-0.477 * *$ \\
\hline & $(0.042)$ & $(0.200)$ & $(0.042)$ & $(0.199)$ & $(0.042)$ & $(0.200)$ & $(0.042)$ & $(0.199)$ & $(0.042)$ & $(0.201)$ \\
\hline \multirow[t]{2}{*}{ Health } & 0.030 & $-0.358 * *$ & 0.032 & $-0.386 * *$ & 0.031 & $-0.381 * *$ & 0.042 & $-0.383 * *$ & 0.044 & $-0.413 * * *$ \\
\hline & $(0.029)$ & $(0.159)$ & $(0.029)$ & $(0.159)$ & $(0.029)$ & $(0.162)$ & $(0.029)$ & $(0.157)$ & $(0.029)$ & $(0.158)$ \\
\hline \multirow{2}{*}{ Services } & 0.020 & $-0.830 * *$ & 0.002 & $-0.872 * *$ & -0.001 & $-0.852 * *$ & 0.022 & $-0.843 * *$ & 0.014 & $-0.894 * *$ \\
\hline & $(0.102)$ & $(0.391)$ & $(0.101)$ & $(0.387)$ & $(0.101)$ & $(0.388)$ & $(0.103)$ & $(0.389)$ & $(0.101)$ & $(0.389)$ \\
\hline \multirow[t]{2}{*}{ Public sector } & $0.215^{* * *}$ & & $0.206^{* * *}$ & & $0.209 * * *$ & & $0.216^{* * *}$ & & $0.212 * * *$ & \\
\hline & $(0.019)$ & & $(0.019)$ & & $(0.019)$ & & (0.019) & & $(0.019)$ & \\
\hline \multirow[t]{2}{*}{ Permanent contract } & $0.156^{* * *}$ & & $0.154 * * *$ & & $0.148 * * *$ & & $0.159 * * *$ & & $0.158 * * *$ & \\
\hline & $(0.017)$ & & $(0.017)$ & & $(0.017)$ & & $(0.017)$ & & $(0.017)$ & \\
\hline \multirow[t]{2}{*}{ Medium firm } & $0.089 * * *$ & & $0.092 * * *$ & & $0.089 * * *$ & & $0.094 * * *$ & & $0.095^{* * *}$ & \\
\hline & $(0.023)$ & & $(0.023)$ & & $(0.022)$ & & $(0.023)$ & & $(0.023)$ & \\
\hline \multirow[t]{2}{*}{ Large firm } & $0.131 * * *$ & & $0.132 * * *$ & & $0.134 * * *$ & & $0.133 * * *$ & & $0.138 * * *$ & \\
\hline & $(0.019)$ & & $(0.019)$ & & $(0.018)$ & & $(0.019)$ & & $(0.019)$ & \\
\hline \multirow[t]{2}{*}{ Father w/ tertiary educ. } & & $-0.213 * *$ & & $-0.219 * *$ & & $-0.211 * *$ & & $-0.234 * * *$ & & $-0.218 * *$ \\
\hline & & $(0.090)$ & & $(0.090)$ & & $(0.091)$ & & $(0.090)$ & & $(0.091)$ \\
\hline \multirow[t]{2}{*}{ Constant } & $1.639 * * *$ & $3.538 * * *$ & $1.541 * * *$ & $3.322 * * *$ & $1.568 * * *$ & $3.461 * * *$ & $1.734 * * *$ & $3.102 * * *$ & $1.550 * * *$ & $3.734 * * *$ \\
\hline & $(0.109)$ & $(0.553)$ & $(0.114)$ & $(0.567)$ & $(0.108)$ & $(0.559)$ & $(0.115)$ & $(0.586)$ & $(0.114)$ & $(0.585)$ \\
\hline Observations & 1997 & & 1997 & & 1997 & & 1997 & & 1997 & \\
\hline Censored Obs. & 152 & & 152 & & 152 & & 152 & & 152 & \\
\hline Sigma & .342 & & .341 & & .339 & & .345 & & .337 & \\
\hline Rho & .691 & & .664 & & .652 & & .665 & & .595 & \\
\hline Lambda & .237 & & .226 & & .221 & & .229 & & .201 & \\
\hline
\end{tabular}

Other controls included: female, age, long program, experience. $* \mathrm{p}<0.10, * * \mathrm{p}<0.05, * * * \mathrm{p}<0.01$ 
Table 5d. Heckman selection with job characteristics

\begin{tabular}{|c|c|c|c|c|c|c|c|c|c|c|}
\hline & \multicolumn{2}{|c|}{ Willingness to question ideas } & \multicolumn{2}{|c|}{ Ability to present } & \multicolumn{2}{|c|}{ Ability to write } & \multicolumn{2}{|c|}{ Foreign language } & \multicolumn{2}{|c|}{ Number of skills } \\
\hline & Ln(wage) & Selection & Ln(wage) & Selection & Ln(wage) & Selection & Ln(wage) & Selection & Ln(wage) & Selection \\
\hline \multirow[t]{2}{*}{ Required level of competence } & $0.033 * * *$ & & $0.027 * * *$ & & $0.034 * * *$ & & $0.015 * * *$ & & $0.012 * * *$ & \\
\hline & $(0.005)$ & & $(0.005)$ & & $(0.005)$ & & $(0.004)$ & & $(0.002)$ & \\
\hline \multirow[t]{2}{*}{ Own level of competence } & 0.002 & 0.018 & -0.003 & 0.003 & $-0.014 *$ & 0.006 & -0.002 & 0.001 & -0.000 & -0.011 \\
\hline & $(0.007)$ & $(0.035)$ & $(0.006)$ & $(0.029)$ & $(0.007)$ & $(0.035)$ & $(0.005)$ & $(0.025)$ & $(0.002)$ & $(0.011)$ \\
\hline \multirow[t]{2}{*}{ Grade secondary educ. } & $0.028 * * *$ & 0.057 & $0.028 * * *$ & 0.048 & $0.031 * * *$ & 0.049 & $0.028 * * *$ & 0.056 & $0.028 * * *$ & 0.048 \\
\hline & $(0.009)$ & $(0.050)$ & $(0.009)$ & $(0.052)$ & $(0.009)$ & $(0.052)$ & $(0.009)$ & $(0.051)$ & $(0.009)$ & $(0.051)$ \\
\hline \multirow[t]{2}{*}{ Education } & $0.079 * *$ & -0.239 & $0.067 * *$ & -0.291 & $0.088 * * *$ & -0.280 & $0.082 * * *$ & -0.251 & $0.083 * * *$ & -0.275 \\
\hline & $(0.031)$ & $(0.183)$ & $(0.031)$ & $(0.183)$ & $(0.031)$ & $(0.184)$ & $(0.031)$ & $(0.184)$ & $(0.031)$ & $(0.187)$ \\
\hline \multirow[t]{2}{*}{ Humanities } & -0.046 & $-0.389 * *$ & -0.046 & $-0.411 * *$ & -0.032 & $-0.401 * *$ & $-0.060^{*}$ & $-0.391 * *$ & -0.040 & $-0.403 * *$ \\
\hline & $(0.034)$ & $(0.172)$ & $(0.035)$ & $(0.175)$ & $(0.035)$ & $(0.175)$ & $(0.035)$ & $(0.174)$ & $(0.034)$ & $(0.176)$ \\
\hline \multirow[t]{2}{*}{ Science\&Maths } & $0.057 * *$ & 0.131 & $0.054 * *$ & 0.116 & $0.056 * *$ & 0.117 & $0.052 * *$ & 0.120 & $0.060 * *$ & 0.116 \\
\hline & $(0.025)$ & $(0.157)$ & $(0.024)$ & $(0.158)$ & $(0.025)$ & $(0.158)$ & $(0.025)$ & $(0.157)$ & $(0.024)$ & $(0.158)$ \\
\hline \multirow[t]{2}{*}{ Engineering } & $0.151 * * *$ & $-0.513 * * *$ & $0.163 * * *$ & $-0.512 * * *$ & $0.163 * * *$ & $-0.511 * * *$ & $0.156 * * *$ & $-0.514 * * *$ & $0.155^{* * *}$ & $-0.501 * * *$ \\
\hline & $(0.027)$ & $(0.128)$ & $(0.027)$ & $(0.129)$ & $(0.027)$ & $(0.129)$ & $(0.027)$ & $(0.128)$ & $(0.027)$ & $(0.130)$ \\
\hline \multirow[t]{2}{*}{ Agriculture \&Vet } & 0.020 & $-0.488 * *$ & 0.031 & $-0.485 * *$ & 0.026 & $-0.490 * *$ & 0.035 & $-0.492 * *$ & 0.033 & $-0.467 * *$ \\
\hline & $(0.042)$ & $(0.200)$ & $(0.042)$ & $(0.202)$ & $(0.042)$ & $(0.202)$ & $(0.042)$ & $(0.200)$ & $(0.042)$ & $(0.203)$ \\
\hline \multirow[t]{2}{*}{ Health } & 0.034 & $-0.394 * *$ & 0.047 & $-0.431 * * *$ & $0.056^{*}$ & $-0.420 * * *$ & 0.041 & $-0.398 * *$ & 0.044 & $-0.424 * * *$ \\
\hline & $(0.029)$ & $(0.159)$ & $(0.029)$ & $(0.158)$ & $(0.029)$ & $(0.160)$ & $(0.029)$ & $(0.160)$ & $(0.029)$ & $(0.160)$ \\
\hline \multirow[t]{2}{*}{ Services } & 0.014 & $-0.879 * *$ & 0.054 & $-0.869 * *$ & 0.052 & $-0.877 * *$ & 0.021 & $-0.865^{* *}$ & 0.052 & $-0.875^{* *}$ \\
\hline & $(0.101)$ & $(0.389)$ & $(0.102)$ & $(0.392)$ & $(0.102)$ & $(0.390)$ & $(0.102)$ & $(0.389)$ & $(0.101)$ & $(0.392)$ \\
\hline \multirow{2}{*}{ Public sector } & $0.213 * * *$ & & $0.211 * * *$ & & $0.205 * * *$ & & $0.216 * * *$ & & $0.221 * * *$ & \\
\hline & $(0.019)$ & & $(0.019)$ & & $(0.019)$ & & $(0.019)$ & & $(0.019)$ & \\
\hline \multirow[t]{2}{*}{ Permanent contract } & $0.156 * * *$ & & $0.155^{* * *}$ & & $0.154 * * *$ & & $0.157 * * *$ & & $0.155^{* * *}$ & \\
\hline & $(0.017)$ & & $(0.017)$ & & $(0.017)$ & & $(0.017)$ & & $(0.017)$ & \\
\hline \multirow{2}{*}{ Medium firm } & $0.097 * * *$ & & $0.099 * * *$ & & $0.100 * * *$ & & $0.096 * * *$ & & $0.096 * * *$ & \\
\hline & $(0.023)$ & & $(0.023)$ & & $(0.023)$ & & $(0.023)$ & & $(0.022)$ & \\
\hline \multirow[t]{2}{*}{ Large firm } & $0.136 * * *$ & & $0.137 * * *$ & & $0.141 * * *$ & & $0.130 * * *$ & & $0.134 * * *$ & \\
\hline & $(0.019)$ & & $(0.019)$ & & $(0.019)$ & & $(0.019)$ & & $(0.018)$ & \\
\hline \multirow[t]{2}{*}{ Father w/ tertiary educ. } & & $-0.227 * *$ & & $-0.226 * *$ & & $-0.224 * *$ & & $-0.231 * *$ & & $-0.216 * *$ \\
\hline & & $(0.090)$ & & $(0.093)$ & & $(0.092)$ & & $(0.092)$ & & $(0.093)$ \\
\hline \multirow[t]{2}{*}{ Constant } & $1.554 * * *$ & $3.247 * * *$ & $1.613 * * *$ & $3.410 * * *$ & $1.598 * * *$ & $3.370 * * *$ & $1.695 * * *$ & $3.357 * * *$ & $1.553 * * *$ & $3.601 * * *$ \\
\hline & $(0.112)$ & $(0.580)$ & $(0.109)$ & $(0.572)$ & $(0.112)$ & $(0.588)$ & $(0.108)$ & $(0.557)$ & $(0.110)$ & $(0.567)$ \\
\hline Observations & 1997 & & 1997 & & 1997 & & 1997 & & 1997 & \\
\hline Censored Obs. & 152 & & 152 & & 152 & & 152 & & 152 & \\
\hline Sigma & .34 & & .334 & & .335 & & .342 & & .332 & \\
\hline Rho & .647 & & .511 & & .551 & & .633 & & .515 & \\
\hline Lambda & .22 & & .171 & & .185 & & .216 & & .171 & \\
\hline
\end{tabular}

Other controls included: female, age, long program, experience. $* \mathrm{p}<0.10, * * \mathrm{p}<0.05, * * * \mathrm{p}<0.01$ 
Table 6. Heckman selection with all competencies included

(1)

(2)

(3)

Ln(wage) selection Ln(wage) selection Ln(wage) Selection

Own level of competencies:

Mastery of own field

\subsection{4}

$(0.009)$

Knowledge of other fields

Analytical thinking

Ability to learn

Ability to negotiate

Work under pressure

Alertness to opportunities.

Ability to coordinate

Ability to use time efficiently

-0.007
$(0.008)$

$0.047 * * *$

(0.009)

$-0.016$

(0.011)

$-0.006$

(0.008)

$-0.009$

(0.008)

$-0.011$

(0.008)

$-0.001$

(0.010)

$-0.030 * * *$

(0.009)

Ability to work with others

$0.019 * *$

(0.009)

Ability to mobilize others

$-0.015^{*}$

(0.009)

Ability to make meaning clear

Ability to assert authority

$-0.008$

(0.010)

$0.036 * * *$

(0.008)

Ability to use computers

Ability to come up with ideas

$-0.014 *$

(0.008)

$-0.000$

(0.011)

0.004

(0.009)

0.007

(0.007)

$-0.010$

(0.009)

0.004

(0.005)

0.006

(0.005)

$-0.013$

(0.044)

0.000

$-0.061$

(0.009)

(0.040)

0.043

(0.042)

0.001

(0.050)

$-0.006$

(0.038)

$-0.061$

(0.040)

$-0.122 * * *$

(0.042)

$-0.043$

(0.050)

$-0.020$

(0.043)

0.169 ***

(0.040)

$-0.086 * *$

(0.044)

$-0.009$

(0.049)

0.033

(0.043)

0.001

(0.043)

$-0.036$

(0.055)

0.032

(0.044)

$0.060^{*}$

(0.034)

0.037

(0.043)

0.000

(0.026)

0.009

(0.021)

$0.100 * * *$

(0.032)

$-0.039$

(0.037)

0.014

(0.027)

$0.111 * * *$

(0.028)

$-0.005$

(0.044)

$0.055^{*}$

(0.030)

0.042

(0.106)

$-0.023$

(0.162)

$-0.200$

(0.161)

0.060

(0.150)

$-0.529 * *$

(0.122)

$-0.457 * *$

(0.190)

$-0.269 *$

(0.140)

$-0.811 * *$

(0.375)

$\underline{\text { Required level of competencies: }}$

Mastery of own field

Knowledge of other fields
$-0.005$

(0.009)

$0.034 * * *$

(0.010)

(0.011)

$-0.011$

(0.008)

$-0.000$

(0.009)

$-0.001$

(0.009)

$-0.013$

(0.010)

$-0.027 * * *$

(0.009)

0.017 *

(0.010)

$-0.018 * *$

(0.009)

$-0.009$

(0.010)

$0.024 * * *$

(0.009)

0.004

(0.009)

0.001

(0.011)

0.002

(0.009)

$-0.000$

(0.008)

$-0.021 * *$

(0.009)

0.003

(0.006)

0.005

(0.005)

$0.079 * *$

(0.032)

$-0.027$

(0.036)

0.019

(0.026)

$0.099 * * *$

(0.027)

$-0.013$

(0.042)

0.030

(0.029)

0.032

(0.103)

$0.019 * * *$

(0.007)

0.001

(0.007)
$-0.015$

$\begin{array}{lll}0.005 & -0.005 & 0.016\end{array}$

$\begin{array}{lll}(0.046) & (0.009) & (0.051)\end{array}$

$\begin{array}{lll}-0.068^{*} & -0.005 & -0.075^{*}\end{array}$

$\begin{array}{lll}(0.041) & (0.008) \quad(0.045)\end{array}$

$\begin{array}{lll}0.041 & 0.029 * * * & 0.051\end{array}$

$\begin{array}{lll}(0.043) & (0.009) & (0.046)\end{array}$

$\begin{array}{lll}0.020 & -0.017 & 0.003\end{array}$

$\begin{array}{lll}(0.052) \quad(0.011) & (0.058)\end{array}$

$\begin{array}{lll}-0.014 & -0.007 & -0.010\end{array}$

$\begin{array}{lll}(0.039) & (0.008) & (0.041)\end{array}$

$\begin{array}{lll}-0.074 * & 0.005 & -0.075\end{array}$

$\begin{array}{lll}(0.041) & (0.009) \quad(0.045)\end{array}$

$-0.115^{* * *} \quad-0.003 \quad-0.116^{* *}$

$\begin{array}{lll}(0.044) & (0.009) \quad(0.046)\end{array}$

$\begin{array}{lll}-0.052 & -0.004 & -0.046\end{array}$

$\begin{array}{lll}(0.051) & (0.010) \quad(0.055)\end{array}$

$\begin{array}{lll}-0.019 & -0.025 * * * & -0.018\end{array}$

$\begin{array}{lll}(0.044) & (0.009) \quad(0.047)\end{array}$

$0.173 * * * \quad 0.017 * \quad 0.190 * * *$

(0.041)

$-0.087 *$

$(0.009)$

$-0.019 * * \quad-0.094 *$

$\begin{array}{lll}(0.045) & (0.009) & (0.049)\end{array}$

$-0.004$

(0.049)

0.037

$-0.010$

(0.010)

$0.017 * *$

$-0.009$

(0.052)

0.029

$(0.008)$

(0.047)

(0.044)

$-0.047$

$-0.001$

0.060

(0.009)

$(0.045)$

(0.057)

0.004

0.034

(0.044)

$0.064 *$

0.001

(0.060)

0.001

0.049

(0.009)

(0.046)

(0.035)

0.041

(0.008)

(0.039)

(0.044)

$-0.014$

0.028

0.002

(0.009)

(0.047)

(0.026)

-0.011

0.005

0.004

(0.029)

(0.022)

(0.005)

(0.025)

$-0.037$

0.051

$-0.256$

(0.169)

(0.031)

(0.189)

$-0.295 *$

$-0.035$

$-0.369 * *$

(0.162)

(0.034)

(0.178)

0.123

$0.051 * *$

0.110

(0.155)

(0.025)

(0.169)

$-0.502 * * *$

$0.142 * * *$

$-0.505 * * *$

(0.126)

(0.026)

(0.135)

$-0.472 * *$

0.001

$-0.490 * *$

(0.192)

(0.041) 
Analytical thinking

Ability to learn

Ability to negotiate

Work under pressure

Alertness to opportunities.

Ability to coordinate

Ability to use time efficiently

Ability to work with others

Ability to mobilize others

Ability to make meaning clear

Ability to assert authority

Ability to use computers

Ability to come up with ideas

Willingness to question ideas

Ability to present

Ability to write reports

Foreign language

Number of skills

Job characteristics:

Public sector

Permanent contract

Medium firm

Large firm

Father w/ tertiary educ.

\section{Constant}

Observations

Censored Obs.

Sigma

Rho

Lambda
$0.015^{*}$
$(0.008)$

$-0.003$

(0.008)

$0.015 * *$

(0.006)

$-0.016^{* *}$

(0.007)

$-0.020 * * *$

(0.007)

0.011

(0.008)

0.005

(0.008)

$-0.001$

(0.007)

0.006

(0.007)

$0.020 * *$

(0.009)

$0.015 * *$

(0.007)

$-0.027 * * *$

(0.007)

0.006

(0.009)

0.002

(0.007)

0.003

(0.007)

$0.018 * *$

(0.007)

0.007

(0.005)

$-0.003$

(0.004)

\begin{tabular}{|c|c|c|c|}
\hline & \multicolumn{3}{|c|}{$\begin{array}{c}0.198 * * * \\
(0.019) \\
0.151 * * * \\
(0.017) \\
0.093 * * * \\
(0.022) \\
0.131 * * * \\
(0.018)\end{array}$} \\
\hline & $\begin{array}{c}-0.192 * * \\
(0.085)\end{array}$ & & $\begin{array}{c}-0.223 * * \\
(0.094)\end{array}$ \\
\hline $\begin{array}{l}1.547 * * * \\
(0.142)\end{array}$ & $\begin{array}{c}2.997 * * * \\
(0.708)\end{array}$ & $\begin{array}{c}1.537 * * * \\
(0.135)\end{array}$ & $\begin{array}{c}3.383 * * * \\
(0.722)\end{array}$ \\
\hline 2154 & & 1997 & \\
\hline 152 & & 152 & \\
\hline .365 & & .327 & \\
\hline .869 & & .633 & \\
\hline .317 & & .207 & \\
\hline
\end{tabular}

0.004

(0.008)

$-0.003$

(0.008)

$0.020 * * *$

(0.006)

$-0.014 * *$

(0.007)

$-0.012 *$

(0.007)

0.010

(0.008)

0.008

(0.008)

$-0.006$

(0.007)

0.006

(0.007)

0.011

(0.008)

0.008

(0.006)

$-0.020 * * *$

(0.007)

0.003

(0.009)

0.008

(0.007)

$-0.002$

(0.007)

$0.015 * *$

(0.007)

0.005

$(0.005)$

$-0.001$

(0.004)

$0.198 * * *$

(0.019)

(0.017)

$0.131 * * *$

Other controls included: female, age, long program, grade secondary education and experience. ${ }^{*} p<0.10,{ }^{* *} p<0.05,{ }^{* * *} p<0.01$ 
Table 7a. Wage of self-employed.

Own field Other fields Analytical thinking Learning Negotiate Under pressure Alertness Coordinate Efficient time use Work with others

\begin{tabular}{|c|c|c|c|c|c|c|c|c|c|c|}
\hline Own level of competence & $\begin{array}{c}0.046 \\
(0.030)\end{array}$ & $\begin{array}{l}0.048 * \\
(0.025)\end{array}$ & $\begin{array}{c}-0.016 \\
(0.024)\end{array}$ & $\begin{array}{l}-0.038 \\
(0.030)\end{array}$ & $\begin{array}{c}0.000 \\
(0.024)\end{array}$ & $\begin{array}{c}0.022 \\
(0.025)\end{array}$ & $\begin{array}{c}0.016 \\
(0.023)\end{array}$ & $\begin{array}{l}-0.007 \\
(0.025)\end{array}$ & $\begin{array}{l}-0.001 \\
(0.025)\end{array}$ & $\begin{array}{l}-0.010 \\
(0.023)\end{array}$ \\
\hline \multirow[t]{2}{*}{ Female } & -0.002 & 0.002 & -0.036 & -0.026 & -0.026 & -0.018 & -0.025 & -0.026 & -0.026 & -0.027 \\
\hline & $(0.066)$ & $(0.065)$ & $(0.066)$ & $(0.064)$ & $(0.065)$ & $(0.065)$ & $(0.064)$ & $(0.065)$ & $(0.065)$ & $(0.065)$ \\
\hline \multirow[t]{2}{*}{ Age } & -0.001 & -0.002 & -0.003 & -0.005 & -0.004 & -0.004 & -0.004 & -0.004 & -0.004 & -0.003 \\
\hline & $(0.013)$ & $(0.013)$ & $(0.013)$ & $(0.013)$ & $(0.013)$ & $(0.013)$ & $(0.013)$ & $(0.013)$ & $(0.014)$ & $(0.014)$ \\
\hline \multirow[t]{2}{*}{ Long program } & $0.236 * * *$ & $0.225 * * *$ & $0.244 * * *$ & $0.241 * * *$ & $0.234 * * *$ & $0.236 * * *$ & $0.235 * * *$ & $0.233 * * *$ & $0.234 * * *$ & $0.230 * * *$ \\
\hline & $(0.067)$ & $(0.067)$ & $(0.069)$ & $(0.067)$ & $(0.068)$ & $(0.067)$ & $(0.068)$ & $(0.068)$ & $(0.068)$ & $(0.068)$ \\
\hline \multirow[t]{2}{*}{ Grade secondary educ. } & -0.002 & -0.008 & -0.003 & -0.003 & -0.006 & -0.006 & -0.004 & -0.006 & -0.006 & -0.005 \\
\hline & $(0.036)$ & $(0.036)$ & $(0.036)$ & $(0.036)$ & $(0.036)$ & $(0.036)$ & $(0.036)$ & $(0.036)$ & $(0.036)$ & $(0.036)$ \\
\hline \multirow[t]{2}{*}{ Experience } & 0.002 & 0.003 & 0.003 & 0.003 & 0.003 & 0.003 & 0.003 & 0.003 & 0.003 & 0.003 \\
\hline & $(0.002)$ & $(0.002)$ & $(0.002)$ & $(0.002)$ & $(0.002)$ & $(0.002)$ & $(0.002)$ & $(0.002)$ & $(0.002)$ & $(0.002)$ \\
\hline \multirow[t]{2}{*}{ Education } & -0.190 & -0.178 & -0.149 & -0.164 & -0.167 & -0.164 & -0.169 & -0.162 & -0.167 & -0.161 \\
\hline & $(0.128)$ & $(0.126)$ & $(0.131)$ & $(0.127)$ & $(0.128)$ & $(0.127)$ & $(0.128)$ & $(0.129)$ & $(0.128)$ & $(0.128)$ \\
\hline \multirow[t]{2}{*}{ Humanities } & 0.105 & 0.137 & 0.149 & 0.157 & 0.141 & 0.136 & 0.145 & 0.145 & 0.142 & 0.140 \\
\hline & $(0.114)$ & $(0.111)$ & $(0.113)$ & $(0.112)$ & $(0.112)$ & $(0.112)$ & $(0.112)$ & $(0.113)$ & $(0.113)$ & $(0.112)$ \\
\hline \multirow[t]{2}{*}{ Science\&Maths } & 0.013 & 0.013 & 0.018 & 0.026 & 0.014 & 0.018 & 0.011 & 0.011 & 0.014 & 0.018 \\
\hline & $(0.114)$ & $(0.113)$ & $(0.115)$ & $(0.114)$ & $(0.116)$ & $(0.114)$ & $(0.114)$ & $(0.115)$ & $(0.115)$ & $(0.115)$ \\
\hline \multirow[t]{2}{*}{ Engineering } & $0.256 * * *$ & $0.275 * * *$ & $0.259 * * *$ & $0.261 * * *$ & $0.257 * * *$ & $0.264 * * *$ & $0.259 * * *$ & $0.257 * * *$ & $0.257 * * *$ & $0.260 * * *$ \\
\hline & $(0.089)$ & $(0.089)$ & $(0.090)$ & $(0.089)$ & $(0.091)$ & $(0.090)$ & $(0.090)$ & $(0.090)$ & $(0.090)$ & $(0.090)$ \\
\hline \multirow[t]{2}{*}{ Agriculture\&Vet } & -0.077 & -0.069 & -0.074 & -0.060 & -0.072 & -0.069 & -0.076 & -0.074 & -0.071 & -0.063 \\
\hline & $(0.126)$ & $(0.125)$ & $(0.127)$ & $(0.127)$ & $(0.127)$ & $(0.127)$ & $(0.127)$ & $(0.127)$ & $(0.128)$ & $(0.128)$ \\
\hline \multirow[t]{2}{*}{ Health } & 0.125 & 0.152 & 0.129 & 0.132 & 0.136 & 0.131 & 0.132 & 0.135 & 0.136 & 0.139 \\
\hline & (0.099) & (0.098) & (0.099) & (0.099) & $(0.099)$ & $(0.099)$ & (0.099) & $(0.099)$ & $(0.100)$ & $(0.099)$ \\
\hline \multirow[t]{2}{*}{ Services } & 0.180 & 0.173 & 0.164 & 0.135 & 0.164 & 0.175 & 0.170 & 0.175 & 0.164 & 0.180 \\
\hline & $(0.207)$ & $(0.206)$ & $(0.208)$ & $(0.209)$ & $(0.209)$ & $(0.208)$ & $(0.208)$ & $(0.212)$ & $(0.209)$ & $(0.212)$ \\
\hline \multirow[t]{2}{*}{ Constant } & $1.586 * * *$ & $1.610 * * *$ & $1.977 * * *$ & $2.150 * * *$ & $1.909 * * *$ & $1.802 * * *$ & $1.842 * * *$ & $1.946 * * *$ & $1.917 * * *$ & $1.948 * * *$ \\
\hline & $(0.484)$ & $(0.459)$ & $(0.450)$ & $(0.475)$ & $(0.458)$ & $(0.455)$ & $(0.449)$ & $(0.456)$ & $(0.477)$ & $(0.448)$ \\
\hline Observations & 152 & 152 & 152 & 152 & 152 & 152 & 152 & 152 & 152 & 152 \\
\hline $\mathrm{R}^{\wedge} 2$ & .245 & .253 & .234 & .241 & .232 & .236 & .234 & .232 & .232 & .233 \\
\hline
\end{tabular}

$* \mathrm{p}<0.10, * * \mathrm{p}<0.05, * * * \mathrm{p}<0.01$ 
Table 7b. Wage of self-employed

\begin{tabular}{|c|c|c|c|c|c|c|c|c|c|c|}
\hline & Mobilize others & Clear meaning & Authority & Computers & Find solutions & Question ideas & Present & Write & Foreign language & N. skills \\
\hline \multirow[t]{2}{*}{ Own level of competence } & -0.008 & -0.011 & 0.020 & 0.036 & 0.006 & 0.004 & 0.016 & $0.044^{*}$ & 0.028 & 0.006 \\
\hline & $(0.022)$ & $(0.026)$ & $(0.023)$ & $(0.024)$ & $(0.028)$ & $(0.024)$ & $(0.020)$ & $(0.025)$ & $(0.017)$ & $(0.007)$ \\
\hline \multirow[t]{2}{*}{ Female } & -0.029 & -0.028 & -0.020 & -0.021 & -0.025 & -0.024 & -0.020 & -0.015 & -0.029 & -0.019 \\
\hline & $(0.065)$ & $(0.065)$ & $(0.065)$ & $(0.064)$ & $(0.065)$ & $(0.066)$ & $(0.065)$ & $(0.064)$ & $(0.064)$ & $(0.065)$ \\
\hline \multirow[t]{2}{*}{ Age } & -0.004 & -0.003 & -0.004 & -0.002 & -0.004 & -0.003 & -0.002 & -0.002 & 0.000 & -0.004 \\
\hline & $(0.014)$ & $(0.014)$ & $(0.013)$ & $(0.013)$ & $(0.014)$ & $(0.014)$ & $(0.014)$ & $(0.013)$ & $(0.014)$ & $(0.013)$ \\
\hline \multirow[t]{2}{*}{ Long program } & $0.234 * * *$ & $0.232 * * *$ & $0.233 * * *$ & $0.226 * * *$ & $0.234 * * *$ & $0.233 * * *$ & $0.232 * * *$ & $0.228 * * *$ & $0.229 * * *$ & $0.232 * * *$ \\
\hline & $(0.068)$ & $(0.068)$ & $(0.067)$ & $(0.067)$ & $(0.068)$ & $(0.068)$ & $(0.067)$ & $(0.067)$ & $(0.067)$ & $(0.067)$ \\
\hline \multirow{2}{*}{ Grade secondary educ. } & -0.006 & -0.004 & -0.007 & -0.008 & -0.007 & -0.007 & -0.007 & -0.014 & -0.010 & -0.007 \\
\hline & $(0.036)$ & $(0.037)$ & $(0.036)$ & $(0.036)$ & $(0.036)$ & $(0.037)$ & $(0.036)$ & $(0.036)$ & $(0.036)$ & $(0.036)$ \\
\hline \multirow[t]{2}{*}{ Experience } & 0.003 & 0.003 & 0.003 & 0.003 & 0.003 & 0.003 & 0.003 & 0.003 & 0.003 & 0.003 \\
\hline & $(0.002)$ & $(0.002)$ & $(0.002)$ & $(0.002)$ & $(0.002)$ & $(0.002)$ & $(0.002)$ & $(0.002)$ & $(0.002)$ & $(0.002)$ \\
\hline \multirow[t]{2}{*}{ Education } & -0.167 & -0.148 & -0.171 & -0.139 & -0.172 & -0.170 & -0.180 & -0.171 & -0.128 & -0.177 \\
\hline & $(0.128)$ & $(0.135)$ & $(0.127)$ & $(0.128)$ & $(0.129)$ & $(0.129)$ & $(0.128)$ & $(0.126)$ & $(0.129)$ & $(0.128)$ \\
\hline \multirow[t]{2}{*}{ Humanities } & 0.142 & 0.152 & 0.133 & 0.144 & 0.142 & 0.142 & 0.129 & 0.144 & 0.096 & 0.135 \\
\hline & $(0.112)$ & $(0.115)$ & $(0.112)$ & $(0.111)$ & $(0.112)$ & $(0.112)$ & $(0.113)$ & $(0.111)$ & $(0.115)$ & $(0.112)$ \\
\hline \multirow{2}{*}{ Science\&Maths } & 0.015 & 0.018 & 0.021 & 0.016 & 0.013 & 0.014 & 0.018 & 0.028 & -0.001 & 0.016 \\
\hline & $(0.115)$ & $(0.115)$ & $(0.115)$ & $(0.114)$ & $(0.115)$ & $(0.115)$ & $(0.114)$ & $(0.114)$ & $(0.114)$ & $(0.114)$ \\
\hline \multirow[t]{2}{*}{ Engineering } & $0.261 * * *$ & $0.257 * * *$ & $0.261 * * *$ & $0.257 * * *$ & $0.257 * * *$ & $0.257 * * *$ & $0.262 * * *$ & $0.261 * * *$ & $0.260 * * *$ & $0.257 * * *$ \\
\hline & $(0.090)$ & $(0.090)$ & $(0.090)$ & $(0.089)$ & $(0.090)$ & $(0.090)$ & $(0.090)$ & $(0.089)$ & $(0.089)$ & $(0.089)$ \\
\hline \multirow[t]{2}{*}{ Agriculture\&Vet } & -0.066 & -0.066 & -0.070 & -0.083 & -0.073 & -0.074 & -0.074 & -0.095 & -0.104 & -0.086 \\
\hline & $(0.128)$ & $(0.128)$ & $(0.127)$ & $(0.126)$ & $(0.127)$ & $(0.128)$ & $(0.127)$ & $(0.126)$ & $(0.128)$ & $(0.128)$ \\
\hline \multirow[t]{2}{*}{ Health } & 0.137 & 0.139 & 0.129 & 0.143 & 0.135 & 0.136 & 0.136 & 0.160 & 0.142 & 0.130 \\
\hline & $(0.099)$ & $(0.099)$ & $(0.099)$ & $(0.098)$ & (0.099) & (0.099) & (0.099) & (0.099) & $(0.098)$ & $(0.099)$ \\
\hline \multirow[t]{2}{*}{ Services } & 0.174 & 0.184 & 0.148 & 0.226 & 0.164 & 0.158 & 0.142 & 0.136 & 0.193 & 0.157 \\
\hline & $(0.210)$ & $(0.213)$ & $(0.209)$ & $(0.211)$ & $(0.208)$ & $(0.212)$ & $(0.210)$ & $(0.207)$ & $(0.207)$ & $(0.208)$ \\
\hline \multirow[t]{2}{*}{ Constant } & $1.967 * * *$ & $1.936 * * *$ & $1.831 * * *$ & $1.645^{* * *}$ & $1.879 * * *$ & $1.885 * * *$ & $1.798 * * *$ & $1.628 * * *$ & $1.708 * * *$ & $1.826 * * *$ \\
\hline & $(0.466)$ & $(0.443)$ & $(0.446)$ & $(0.469)$ & $(0.459)$ & $(0.461)$ & $(0.459)$ & $(0.463)$ & $(0.453)$ & $(0.449)$ \\
\hline Observations & 152 & 152 & 152 & 152 & 152 & 152 & 152 & 152 & 152 & 152 \\
\hline $\mathrm{R}^{\wedge} 2$ & .233 & .233 & .236 & .244 & .232 & .232 & .236 & .248 & .246 & .236 \\
\hline
\end{tabular}

$* \mathrm{p}<0.10, * * \mathrm{p}<0.05, * * * \mathrm{p}<0.01$ 